\title{
Phenotypic Study on the Bacterial Isolates from Equine with Respiratory Disorders regarding Antimicrobial Drug Resistance
}

\author{
M. Fawzy Nehal ${ }^{1}$, M. Osman Kamelia ${ }^{2}$, N. F. Azza ${ }^{1}$, R. A. Abd Elmawgoud Shaimaa ${ }^{1}$, S.A. El Shafii Soumaya ${ }^{1 *}$, \\ M. A. Shahein ${ }^{1}$ and E. M. Ibraheem ${ }^{1}$ \\ ${ }^{1}$ Animal Health Research Institute; Equine Bacterial Diseases Unit, Giza, Egypt \\ ${ }^{2}$ Faculty of Veterinary Medicine, Cairo University, Egypt \\ *Corresponding author's Email: Dr.soumayaelshafii@yahoo.com; (D)RCiD: 0000-0003-1071-8377
}

\begin{abstract}
Upper respiratory tract infection and pneumonia in foals are primarily caused by a bacterial infection. Gramnegative bacteria are commonly found in neonatal pneumonia although gram-positive and mixed infections could be accompanied. The current study aimed to detect the different pathogens causing respiratory disorders in the equine, describe the antimicrobial resistance in these pathogens, and determine the types of antimicrobial isolates. A total of 203 different samples were collected from 42 horse foals, 5 adult horses, and 4 donkey foals from June 2019 to April 2020. All samples were subjected to bacteriology analysis and isolated bacteria were analyzed using susceptibility test for different antibacterial agents. The findings indicated that $38(74.5 \%)$ animals were positive for the isolation of bacteria causing respiratory disorders. The most predominant isolates were Klebsiella pneumoniae subsp. Pneumoniae followed by Staphylococcus aureus, Streptococcus equi, Pseudomonas aeruginosa, Streptococcus zooepidemicus, Proteus mirabilis, Rhodococcus equi, Stenotrophomonas maltophilia, and Streptococcus mitis. Stenotrophomonas maltophilia is isolated from all organs, including the lungs. All K. pneumoniae isolates were sensitive to lomefloxacin, cefotaxime, meropenem, enrofloxacin, neomycin, and chloramphenicol. The Pseudomonas aerugenosa (P. aeruginosa) is sensitive to aztreonam and $20 \%$ of isolates sensitive to Piperacillintazobactam. All Proteus mirabilis were sensitive to ampicillin-sulbactam, piperacillin-tazobactam, and cefoperazone. Stenotrophomonas maltophilia was only sensitive to oxytetracycline and lomefloxacin. Staphylococcus aureus was susceptible to Piperacillin-tazobactam (50\%), 25\% to lomefloxacin; Streptococcus equi were sensitive to vancomycin $33.3 \%$ while $16.7 \%$ to erythromycin and doxycycline, Streptococcus zooepidemicus $(100 \%)$ were sensitive to cefotaxime, meropenem, and doxycycline. All isolates of Enterococcus species were sensitive to penicillin, piperacillin-tazobactam, and lomefloxacin. Moreover, Rhodococcus equi (one isolate) was only sensitive to clarithromycin. The antimicrobial susceptibility test illustrated the presence of multidrug-resistant and pan-drug resistant isolates which proved the indiscriminate and extensive use of antibiotics. In conclusion, resistance monitoring data and risk assessment identified several direct and/or indirect predisposing factors to be potentially associated with MDR development in the equine health sector of Egypt. The predisposing factors may be attributed to insufficient veterinary healthcare, monitoring, and regulatory services, in addition to the intervention of animal health service providers, and/ or farmers' lack of knowledge about drugs. The misuse and overuse of antibiotics have led to the evolution of antibiotic-resistant bacteria in equine in Egypt.
\end{abstract}

Keywords: Antimicrobial agents, Klebsiella pneumoniae, Streptococcus zooepidemicus.

\section{INTRODUCTION}

Substantial morbidity and mortality in foals are commonly due to lower and upper respiratory tract infections that is attributed to the interactions between innate immunologic factors and management risk factors (Galvin and Corley 2010). Neonatal pneumonia is commonly caused by Gram-negative bacteria, although Gram-positive and mixed infections do occur. The development of pneumonia can be complex in the foal as it can be caused by multiple organisms-viruses, bacteria, and even internal parasites (Léguillette et al., 2002).

Pneumonia in foals is primarily caused by a bacterial infection and among all isolates, Streptococcus zooepidemicus and Rhodococcus equi are the most important Gram-positive bacteria. These organisms can be obtained from pure culture or a pleurimicrobial infection. Several other aerobic bacterial species may also occur, including, Actinobacillus spp, Bordetella bronchiseptica, Escherichia coli, Klebsiella pneumoniae, Pasteurella spp, Pseudomonas spp, Salmonella spp., and Staphylococcus spp. (Welsh, 1984). Klebsiella spp. is concerned as a common cause of bacterial pneumonia in horses, but few reports describe the clinical presentation and disease progression (Estell et al., 2016). Strangles is a highly contagious disease caused by the abscess-forming bacteria Streptococcus equi, mainly foals, and horses of any age can also be infected. It seems to cause severe and economically important respiratory disease in horses (Erol et al., 2012; Rush, 2014). One Health (OH) is a vital conceptualization when the intervention that occurs 
between humans, animals, and the environment is considered. The horses' footprint on the well-being of the environment and humans forces the incorporation of the horse in any roadmap to achieve OH (Lönker et al., 2020). Antimicrobial resistance in equine medicine has received relatively limited attention which encourages individuals to indulge in this endeavor to throw light on the situation of microbial resistance in the bacterial community allocated in the respiratory tract of equines.

The aim of this study was to detect the rate of different pathogens causing respiratory disorders in equine and describe the rate of antimicrobial resistance in pathogens, and to determine the type of antimicrobial isolates.

\section{MATERIALS AND METHODS}

A database search was performed of submissions to Equine Bacterial Diseases Unit (EBDU) within time interval June 2019 to April 2020 for the bacterial culture of samples from foals, adults, and donkeys (Table 1). Samples were enriched on buffer peptone water and incubated at 37C for 18-24 hours. The enriched samples were cultured on duplicated plates blood agar and staph strep media with strep supplement and 5\% sheep blood (UK standard, 2014a). Also, the enriched samples were cultured on mannitol media or Baird Barker media, and plates incubated at $37^{\circ} \mathrm{C}$ for 24 hours (UK standard, 2014b), and on Tinsdale media at $37^{\circ} \mathrm{C}$ for $24-72$ hours aerobically (UK standard, 2014c). Small Colonies showed $\beta$ hemolysis or $\alpha$ hemolysis, which were examined for catalase test and oxidase test, golden yellow on mannitol or black colonies with hallow zone on Baird Barker. Non-hemolytic colonies and Tinsdale agar showed small dark brown colonies. Furthermore, enrichment samples were cultured on blood agar, MacConkey agar (UK standard, 2014d), and pseudomonas agar (CN media, UK standard, 2015). All suspected colonies were further biochemical identified using S.R.O. GP24 and S.R.O. GN24 kits (diagnostics.S.R.O. ${ }^{\mathrm{TM}}$ ).

\section{Susceptibility test for different isolates against antimicrobial agents}

The type, symbol, and concentration of antimicrobial agent used were illustrated in supplementary Table 2. Each culture was cultured onto a non-inhibitory agar medium. After incubation at $35^{\circ} \mathrm{C}$ overnight, four or five well-isolated colonies were selected and transferred to Mueller-Hinton broth and vortex thoroughly, incubated the broth at $35^{\circ} \mathrm{C}$, and then adjusted the turbidity ( $0.5 \mathrm{McFarland}$ standard tube). The procedure continued by using a sterile cotton swab, dipping into the suspension, and culturing over the entire surface of the medium, and rotating the plate approximately 60 degrees after each application. This procedure was repeated three times to ensure an even distribution of the inoculum, CLSI, 2012). The antimicrobial discs were applied to the plates and incubated at $35^{\circ} \mathrm{C}$ for 16 to 18 hours. The diameter of the zones of complete inhibition was measured. Interpretation of results was recorded according to CLSI (2017). Pareto chart was used to demonstrate the contribution of each type of bacteria in respiratory infections. It was conducted using QI Macros software that has been loaded to the startup directory of Microsoft Office Excel 2013.

Table 1. Type and numbers of samples collected from private farms in Egypt from June 2019 to April 2020

\begin{tabular}{|c|c|c|c|c|}
\hline $\begin{array}{l}\text { Period of } \\
\text { collection }\end{array}$ & $\begin{array}{c}\text { Type of } \\
\text { animals }\end{array}$ & $\begin{array}{c}\text { Total } \\
\text { number of } \\
\text { animals }\end{array}$ & $\begin{array}{l}\text { Type of } \\
\text { samples }\end{array}$ & $\begin{array}{c}\text { Number of } \\
\text { each type of } \\
\text { samples }\end{array}$ \\
\hline \multirow{6}{*}{$6-12 / 2019$} & \multirow{3}{*}{$\begin{array}{l}\text { Foals } \\
\text { (20 days- } \\
\text { 3years) }\end{array}$} & \multirow{2}{*}{9} & Nasal swabs & 9 \\
\hline & & & Feces & 1 \\
\hline & & 12 & $\begin{array}{l}\text { Internal } \\
\text { organs* }\end{array}$ & 81 \\
\hline & \multirow{3}{*}{ Adults } & \multirow{3}{*}{2} & Nasal swabs & - \\
\hline & & & Feces & - \\
\hline & & & $\begin{array}{l}\text { Internal } \\
\text { organs* }\end{array}$ & 14 \\
\hline Subtotal & & 23 & & 105 \\
\hline \multirow{6}{*}{$1-4 / 2020$} & \multirow{3}{*}{$\begin{array}{c}\text { Foals }(20 \\
\text { days- } \\
\text { 3years }\end{array}$} & \multirow{2}{*}{10} & Nasal swabs & 10 \\
\hline & & & feces & 10 \\
\hline & & 11 & $\begin{array}{l}\text { Internal } \\
\text { organs* }\end{array}$ & 52 \\
\hline & \multirow{3}{*}{ Adults } & \multirow{3}{*}{3} & Nasal swabs & - \\
\hline & & & feces & - \\
\hline & & & $\begin{array}{l}\text { Internal } \\
\text { organs* }\end{array}$ & 18 \\
\hline Subtotal & & 24 & & 90 \\
\hline \multirow{2}{*}{$3 / 2020$} & \multirow{2}{*}{$\begin{array}{l}\text { Donkey's } \\
\text { foal }\end{array}$} & \multirow{2}{*}{4} & Nasal swabs & 4 \\
\hline & & & Feces & 4 \\
\hline Subtotal & & 4 & & 8 \\
\hline Total & & 51 & & 203 \\
\hline
\end{tabular}

Table 2. List of antimicrobial disks used for antibiotic sensitivity test

\begin{tabular}{|c|c|c|c|}
\hline Serial & Antimicrobial agents & Symbol & $\begin{array}{c}\text { Concentration } \\
(\mu \mathrm{g})\end{array}$ \\
\hline 1 & Penicillin & $\mathrm{P}$ & 10 \\
\hline 2 & Oxacillin & $\mathrm{OX}$ & 1 \\
\hline 3 & Ampicillin & AMP & 10 \\
\hline 4 & Ampicillin-sulbactam & SAM & 20 \\
\hline 5 & Ampicillin-clavulanic acid & AMC & 30 \\
\hline 6 & Piperacillin-tazobactam & $\mathrm{TZP}$ & 110 \\
\hline 7 & Cephalexin & $\mathrm{CL}$ & 30 \\
\hline 8 & Cephradine & $\mathrm{CE}$ & 30 \\
\hline 9 & Cefotaxime & CTX & 30 \\
\hline 10 & Cefoperazone & CFP & 75 \\
\hline 11 & Cefquinome & CEQ & 30 \\
\hline 12 & Meropenem & MEM & 10 \\
\hline 13 & Aztreonam & ATM & 300 \\
\hline 14 & Clarithromycin & CLR & 10 \\
\hline 15 & Erythromycin & $\mathrm{E}$ & 15 \\
\hline 16 & Oxytetracycline & OT & 30 \\
\hline 17 & Chloramphenicol & $\mathrm{C}$ & 30 \\
\hline 18 & Norfloxacin & NO & 10 \\
\hline 19 & Ofloxacin & OFX & 5 \\
\hline 20 & Lomefloxacin & LOM & 10 \\
\hline 21 & kanamycin & $\mathrm{K}$ & 30 \\
\hline 22 & Novobiocin & NV & 30 \\
\hline 23 & Streptomycin & $\mathrm{S}$ & 10 \\
\hline 24 & Neomycin & $\mathrm{N}$ & 10 \\
\hline 25 & Amikacin & AK & 30 \\
\hline 26 & Linezolid & LZD & 30 \\
\hline 27 & Clindamycin & DA & 2 \\
\hline 28 & Vancomycin & VA & 30 \\
\hline 30 & Amoxicillin- clavulanic acid & $\begin{array}{l}\text { Amox- } \\
\text { clav }\end{array}$ & 30 \\
\hline 31 & Doxycycline & $\mathrm{D}$ & 30 \\
\hline
\end{tabular}


Recently, an obvious growing interest in equine breeding and industry in Egypt has been observed, which has a great impact on the healthcare of horses as a whole, and particularly on their respiratory infection.

Pneumonia in equine is most frequently caused by Gram-positive bacteria which may be accompanied by Gramnegative (Estell et al., 2016). Out of 51 horses (203 samples), 38 (74.5\%) animals were positive for isolation of bacteria causing respiratory disorders. The rates of different bacteria isolated from different samples in foals and adults are illustrated in Table 3. As can be seen, 36 isolates were obtained (17.7\%) which was less than the obtained of ToombsRuane et al. $(2015,63 \%)$. These different results may be attributed to the different environmental or climatic conditions. About $23.8 \%$ of the microorganisms were isolated from samples collected during the period June 2019 to December 2019 and $12.2 \%$ collected during the period January 2020 to April 2020. Samples of diseased donkey's foals showed no bacteria. The decrease in the isolation rate may be attributed to slight care of the hygienic management (Saastamoinen et al., 2015).

Klebsiella pneumoniae (K. pneumoniae) showed the highest rate of isolation regarding internal organs (26.3\%, Table 4), followed by Staphylococcus aureus (S. aureus), Streptococcus equi subsp. Equi (S. equi subsp. Equi), and Pseudomonas aeruginosa (P. aeruginosa) $(10.5 \%, 4.5 \%$, and 3.8\% respectively). Also, Proteus mirabilis (P. mirabilis) and Streptococcus equi subsp. zooepidemicus (S. zooepidemicus) were isolated at the same rate of $2.3 \%$. Nasal samples of foals showed one isolate $S$. aureus and one isolate of Rhodococcus equi $(R$. equi) isolated from fecal samples. In adult horses, only Enterococcus species isolated from internal organs had a rate of 6.2\% (Table 5). Klebsiella spp are a common cause of bacterial pneumonia but cases are not well-described in the literature, as Estell et al. (2016) stated that mixed infection (polymicrobic infection) is more common in older foals, in which S. zooepidemicus is the most predominant, followed by Actinobacillus suis, and Pasteurella spp. The obtained results of E. coli, Klebsiella pneumoniae were on the contrary with Wood et al. (2005) who found that $S$. zooepidemicus and S. pneumoniae are the most common ones followed by Actinobacillus, Pasteurella, and Mycoplasma equirhinis.

Stenotrophomonas maltophilia (S. maltophilia) is isolated from all organs, including the lung, for the first time in Egypt . Recently, S. maltophilia is being recorded as a human nosocomial infection causing pneumonia with increasing incidence and has not previously been associated with lower airway disease in the horse. However, Winther et al. (2009) reported the clinical findings, laboratory diagnosis, and response to treatment of seven cases of respiratory infection with S. maltophilia in horses.

Table 6 and Figures 1 and 2 showed the rate of single and mixed infection in dead animals, where 5 animals showed mixed infection with $K$. pneumoniae and S. aureus (13.1\%), also S. aureus with Ps. aeruginosa was a mixed infection in $7.9 \%$ of cases. The K. pneumoniae indicated the highest rate of single infection (26.3\%). Stenotrophomonas maltophilia (2.6\%) as it isolated from all organs, including lung, is isolated in Egypt for the first time. These obtained results were in agreement with those reported by Wilson (2001).

Antimicrobial agent's action occurs by interrupting specific metabolic functions within bacterial cells. There are four primary targets for antimicrobial action, including disruption of cell wall synthesis, inhibition of DNA/RNA synthesis, inhibition of protein biosynthesis, or interference with a crucial metabolic pathway (Roberts, 2005). There has been a scarcity in the studies investigating the antimicrobial resistance profile in the bacteria that have been isolated from the respiratory tract of horses (Johns and Adams, 2015; Álvarez-Narváez et al., 2020; Lönker et al., 2020).

The $K$. pneumoniae. isolates were sensitive to lomefloxacin, cefotaxime, meropenem, enrofloxacin, neomycin, and chloramphenicol $(15.4 \%, 13.3 \%, 13.3 \%, 6.7 \%, 6.7 \%$, and 6.7\%, respectively, Table 7). Fluoroquinolones are predominantly active against Gram-negative aerobes, including Enterobacteriaceae and Pseudomonas aeruginosa, against Mycoplasma spp., Rickettsia spp., and Ehrlichia spp. They have limited Gram-positive coverage, except for many Staphylococcus spp. (Haggett and Wilson, 2008). Enrofloxacin is the only fluoroquinolone presently in clinical use in horses. Although different doses have been reported in the literature for other fluoroquinolones, there is a lack of reliable data (Bousquet-Melou et al., 2002; Davis et al., 2006; Fernandez-Varon et al., 2006).

The $P$. aeruginosa is sensitive to aztreonam (100\%) and $20 \%$ of isolates sensitive to Piperacillin-tazobactam. The monobactams do not have any activity against Gram-positives or anaerobic bacteria. However, they are highly effective against certain Gram-negative bacteria, especially the enteric Gram-negative rods and can be used for Pseudomonas aeruginosa (Chirality, 2012). All Proteus mirabilis (3 isolates) were sensitive to ampicillin-sulbactam, piperacillintazobactam, and cefoperazone (100\%). Only, $33.3 \%$ of isolates were sensitive to enrofloxacin, Stenotrophomonas maltophilia (one isolate) was sensitive to oxytetracycline (Table 7). These results were in accordance with O'Hara et al. (2000) and Deredjian et al. (2016). As can be seen in Table 7, S. aureus was susceptible to Piperacillin-tazobactam $(50 \%)$ and $25 \%$ to lomefloxacin. It was recorded that the bactericidal activity of piperacillin/tazobactam was noticed 1 hour after drug administration for S. aureus, E. coli, and P. aeruginosa (Lemmen et al., 2004). Moreovr, it was found that $S$. equi (causing strangles) was sensitive to doxycycline and erythromycin (16.7\%). S. zooepidemicus was sensitive to cefotaxime, meropenem, and doxycycline (100\%), which supported the findings of Lemmen et al. (2004). 
$R$. equi (one isolate) was sensitive only to Clarithromycin (Table 7). Pneumonia caused by $R$. equi is a major health problem for equine industries on a worldwide basis. A combination of macrolide with rifampin is recommended for the treatment of infection caused by $R$. equi (according to the in-vitro activity) when there are no highly effective preventatives (Gigue're et al., 2011). Heatmap analysis showed the intensity of antibiotic resistance of different isolates based on the percentage of resistance (Figure 3). Each row indicates the type of isolate and each column represents the type of antimicrobial agents most of which showed 100\% resistance. The phenotypic resistance pattern, prevalence, and diversity of the four Gram-ve bacteria species K. pneumoniae, P. aeruginosa, P. mirabilis, and Stenotrophomonas maltophilia isolates are recorded in Table 8. They were tested for their resistance phenotypic profile against 25 antibiotics representing 9 classes. They were resistant to the 15 antibiotics. Moreover, the five Gram-positive bacteria species isolated from the respiratory tract ( $S$. aureus, S. equi, and S. zooepidemicus), feces Enterococcus spp., and one isolate of $R$. equi were tested for their phenotypic resistance patterns against 27 antibiotics representing 11 classes (Table 9).

This diversity of Gram-negative bacteria and Gram-positive bacteria isolated from the respiratory tract reflect the capacity of AMR revealed the indiscriminate and extensive use of antibiotics which has led to the emergence and extent spread of resistant pathogenic bacteria (Wolska et al., 2012; Garza-Cervantes et al., 2020). Highly resistant Gramnegative bacteria were Pseudomonas aeruginosa and Klebsiella pneumoniae have become very difficult to treat pathogens (Boucher et al., 2009) and are, therefore, considered as the ESKAPE pathogens (Pendleton et al., 2013), including some Gram-positive bacteria, such as Staphylococcus, S.equi, and S.zooepidemicus as well as Enterococcus species (Coates et al., 2002; Smith and Romesberg, 2007; Hegreness et al., 2008).

In the present study, 11 isolates of $K$. pneumoneae were typed as multidrug resistance (MDR) and 4 isolates were pan-drug resistance (PDR), all isolates of P. aeruginosa were PDR while all isolates of P. mirabilis and S. maltophilia were MDR (Table 10). While all isolates of Gram-positive isolates were PDR except the two isolates of $S$. zooepidemicus which were MDR (Table 11). Antibiotic resistance (El Zowalaty et al., 2015; Magiorakos et al., 2012) is classified into MDR which is not susceptible to at least one representative from each of three categories of selected antimicrobial compound families (El Zowalaty et al., 2015; Fodor et al., 2020). Extreme or extensively drug-resistant (XDR) is not susceptible to at least a single representative of all but very few categories of antimicrobial compound families. The PDR is not susceptible to any of the tested or empirical representatives of all known antimicrobial compound families (El Zowalaty et al., 2015).

The MDR and PDR isolates are inconsistent in medical literature, disqualifying reliable comparison of data. In order to reach a standardized definition, we applied the multidrug resistance definition from human medicine (Magiorakos et al., 2012). This adaption was limited by the unattainability of certain susceptibility results and differing antimicrobial agents in human and veterinary medicine. Therefore, the establishment of a standard definition of MDR bacteria in veterinary medicine should be supported.

Table 3. Rate of different bacteria isolated from different samples collected from private equine farms during the period from June 2019 to April 2020

\begin{tabular}{|c|c|c|c|c|c|c|c|}
\hline \multirow[b]{2}{*}{$\begin{array}{l}\text { Period of sample } \\
\text { collected }\end{array}$} & \multirow[b]{2}{*}{$\begin{array}{l}\text { Number of } \\
\text { animals }\end{array}$} & \multirow[b]{2}{*}{ Age of horses } & \multirow[b]{2}{*}{ Type of samples } & \multirow[b]{2}{*}{$\begin{array}{c}\text { Total Number } \\
\text { of samples }\end{array}$} & \multicolumn{3}{|c|}{ Results } \\
\hline & & & & & $\begin{array}{c}\text { Number of } \\
\text { positive } \\
\text { samples }\end{array}$ & $\begin{array}{c}\text { Number of } \\
\text { negative samples }\end{array}$ & $\begin{array}{c}\% \text { of positive } \\
\text { results }\end{array}$ \\
\hline \multirow{6}{*}{$6-12-2019$} & \multirow{3}{*}{21} & \multirow{3}{*}{$\begin{array}{l}\text { Foals } \\
\text { ( } 20 \text { days- } 3 \\
\text { years) }\end{array}$} & Internal organs & 81 & 18 & 63 & 22.2 \\
\hline & & & Nasal & 9 & 0 & 9 & 0 \\
\hline & & & Fecal & 1 & 1 & 0 & 0 \\
\hline & \multirow{3}{*}{2} & \multirow{3}{*}{$\begin{array}{l}\text { Adults over } 3 \\
\text { years }\end{array}$} & Internal organs & 14 & 6 & 8 & 42.9 \\
\hline & & & Nasal & 0 & 0 & 0 & 0 \\
\hline & & & Fecal & 0 & 0 & 0 & 0 \\
\hline Subtotal & 23 & & & 105 & 25 & 80 & $23.8 \%$ \\
\hline \multirow{6}{*}{$1-4-2020$} & \multirow{3}{*}{11} & \multirow{3}{*}{ Foals } & Internal organs & 52 & 11 & 41 & 21.2 \\
\hline & & & nasal & 10 & 0 & 10 & 0 \\
\hline & & & Fecal & 10 & 0 & 10 & \\
\hline & \multirow{3}{*}{3} & \multirow{3}{*}{ Adults } & Internal organs & 18 & 0 & 18 & 0 \\
\hline & & & Nasal & - & - & - & \\
\hline & & & Fecal & - & - & - & \\
\hline Subtotal & 14 & & & 90 & 11 & 73 & $12.2 \%$ \\
\hline \multirow{6}{*}{$1-4 / 2020$} & \multirow{6}{*}{4} & \multirow{3}{*}{ Donkey`s foal } & Internal organs & 0 & 0 & 0 & 0 \\
\hline & & & Nasal & 4 & 0 & 4 & 0 \\
\hline & & & Fecal & 4 & 0 & 4 & 0 \\
\hline & & \multirow{3}{*}{ Adult donkeys } & Internal organs & 0 & 0 & 0 & 0 \\
\hline & & & Nasal & 0 & 0 & 0 & 0 \\
\hline & & & Fecal & 0 & 0 & 0 & 0 \\
\hline Subtotal & & & & 8 & 0 & 8 & \\
\hline Total & 50 & - & - & 203 & 36 & 161 & $17.7 \%$ \\
\hline
\end{tabular}

* Percentage calculated according to total number of each type of samples 
Table 4. Number and type of different bacteria isolated from different samples of foals during the period from June 2019 to April 2020.

\begin{tabular}{|c|c|c|c|c|}
\hline Type of samples & $\begin{array}{c}\text { Number of } \\
\text { samples }\end{array}$ & Type of isolated bacteria & $\begin{array}{l}\text { Number of } \\
\text { isolated } \\
\text { organisms }\end{array}$ & Percentage $^{*}$ \\
\hline Nasal swabs & 23 & Staph. aureus & 1 & 4.3 \\
\hline Fecal swabs & 15 & Rhodococcus equi & 1 & 6.7 \\
\hline \multirow{8}{*}{ Internal organs } & \multirow{8}{*}{133} & Stenotrophomonas maltophilia & 1 & 0.8 \\
\hline & & Staph. aureus & 8 & 6.01 \\
\hline & & Streptococcus. zooepidemicus & 3 & 2.3 \\
\hline & & Streptococcus equi subsp. equi & 6 & 4.5 \\
\hline & & Streptococcus mitis & 1 & 0.8 \\
\hline & & Pseudomonas aeruginosa & 5 & 3.8 \\
\hline & & Klebsiella pneumoniae & 15 & 11.2 \\
\hline & & Proteus mirabilis & 3 & 2.3 \\
\hline Total & 171 & - & 44 & 61.9 \\
\hline
\end{tabular}

* Percentage calculated according to the total number of samples

Table 5. Number of different bacteria isolated from different samples in adult horses' equine during the period from June 2019 to April 2020.

\begin{tabular}{|c|c|c|c|c|}
\hline Type of samples & $\begin{array}{l}\text { Number of } \\
\text { samples }\end{array}$ & $\begin{array}{c}\text { Type of isolated } \\
\text { bacteria }\end{array}$ & $\begin{array}{c}\text { Number of isolated } \\
\text { organisms }\end{array}$ & Percentage $^{*}$ \\
\hline Internal organs & 32 & Enterococcus spp. & 2 & $6.2 \%$ \\
\hline Total & 32 & - & 2 & $6.2 \%$ \\
\hline
\end{tabular}

* Percentage calculated according to the total number of samples

Table 6. Rate of isolated bacteria among infected horses during the period from June 2019 to April 2020.

\begin{tabular}{|c|c|c|c|c|c|c|c|}
\hline Type of bacteria & $\begin{array}{l}\text { Type of } \\
\text { positive organs }\end{array}$ & $\begin{array}{l}\text { Number of } \\
\text { isolates in } \\
\text { IO of foals }\end{array}$ & $\begin{array}{c}\text { Number } \\
\text { of isolates } \\
\text { in Fecal } \\
\text { swab }\end{array}$ & $\begin{array}{c}\text { Number } \\
\text { of isolates } \\
\text { in Nasal } \\
\text { swab } \\
\end{array}$ & $\begin{array}{c}\text { Number } \\
\text { of isolates } \\
\text { in IO of } \\
\text { adults }\end{array}$ & $\begin{array}{c}\text { Number } \\
\text { of positive } \\
\text { animals }\end{array}$ & $\begin{array}{c}\text { Rate of } \\
\text { bacterial } \\
\text { isolates* }\end{array}$ \\
\hline Rhodococcus equi & - & 0 & 1 & 0 & 0 & 1 & $2.6 \%$ \\
\hline Klebsiella pneumoniae & All organs & 10 & 0 & 0 & 0 & 10 & $26.3 \%$ \\
\hline Staphylococcus aureus & All organs & 3 & 0 & 1 & 0 & 4 & $10.5 \%$ \\
\hline $\begin{array}{l}\text { Klebsiella pneumoniae + } \\
\text { Staphylococcus aureus }\end{array}$ & Lung + trachea & 5 & 0 & 0 & 0 & 5 & $13.1 \%$ \\
\hline Streptococcus equi subsp. equi & Lung + trachea & 3 & 0 & 0 & 0 & 3 & $7.9 \%$ \\
\hline $\begin{array}{l}\text { Streptococcus equi }+ \text { Pseudomonas } \\
\text { aeruginosa }\end{array}$ & All organs & 3 & 0 & 0 & 0 & 3 & $7.9 \%$ \\
\hline Streptococcus zooepidemicus & Lung & 3 & 0 & 0 & 0 & 3 & $7.9 \%$ \\
\hline Pseudomonas aeruginosa & All organs & 2 & 0 & 0 & 0 & 2 & \\
\hline Streptococcus mitis & $\begin{array}{l}\text { Lung, liver, } \\
\text { spleen }\end{array}$ & 1 & 0 & 0 & 0 & 1 & $2.6 \%$ \\
\hline Stenotrophomonas maltophilia & All organs & 1 & 0 & 0 & 0 & 1 & $2.6 \%$ \\
\hline Proteus mirabilis & All organs & 3 & 0 & 0 & 0 & 3 & $7.9 \%$ \\
\hline Enterococcus species & All organs & 0 & 0 & 0 & 2 & 2 & $5.3 \%$ \\
\hline Total & & 34 & 1 & 1 & 2 & 38 & 89.5 \\
\hline
\end{tabular}

* Rate of bacterial isolates was calculated according to the total Number of animals (38), IO: Internal organs 
Table 7. Susceptibility antimicrobial agents for different bacterial isolates.

\begin{tabular}{|c|c|c|c|c|c|c|c|c|c|c|c|c|c|c|c|c|c|c|}
\hline \multirow{3}{*}{ Antimicrobial agents } & \multicolumn{8}{|c|}{ Gram negative bacteria } & \multicolumn{10}{|c|}{ Gram negative } \\
\hline & \multicolumn{2}{|c|}{$\begin{array}{c}\text { Klebsiella. } \\
\text { pneumoniae }\end{array}$} & \multicolumn{2}{|c|}{$\begin{array}{c}\text { Pseudomonas } \\
\text { aeruginosa }\end{array}$} & \multicolumn{2}{|c|}{ Proteus mirabilis } & \multicolumn{2}{|c|}{$\begin{array}{c}\begin{array}{c}\text { Stenotrophomon } \\
\text { as maltophilia }\end{array} \\
\end{array}$} & \multicolumn{2}{|c|}{$\begin{array}{c}\text { Staphylococcus } \\
\text { aureus }\end{array}$} & \multicolumn{2}{|c|}{$\begin{array}{c}\text { Streptococcus } \\
\text { equi }\end{array}$} & \multicolumn{2}{|c|}{$\begin{array}{c}\text { Streptococcus } \\
\text { zooepidemicus }\end{array}$} & \multicolumn{2}{|c|}{$\begin{array}{c}\text { Enterococcus } \\
\text { species }\end{array}$} & \multicolumn{2}{|c|}{$\begin{array}{c}\text { Rhodococcus } \\
\text { equi }\end{array}$} \\
\hline & No. & $\%$ & No. & $\%$ & No. & $\%$ & No. & & No. & $\%$ & No. & $\%$ & No. & $\%$ & No. & $\%$ & No. & $\%$ \\
\hline \multicolumn{19}{|l|}{$\beta$-lactam } \\
\hline \multicolumn{19}{|l|}{ Penicillins } \\
\hline Penicillin & 0 & 0 & 0 & 0 & 0 & 0 & 0 & 0 & 0 & 0 & 0 & 0 & 0 & 0 & 2 & 100 & 0 & 0 \\
\hline Oxacillin & . & - & 0 & 0 & 0 & 0 & 0 & 0 & 0 & 0 & 0 & 0 & - & - & 0 & 0 & 0 & 0 \\
\hline Ampicillin & 0 & 0 & 0 & 0 & 0 & 0 & 0 & 0 & 0 & 0 & 0 & 0 & 0 & 0 & 0 & 0 & 0 & 0 \\
\hline \multicolumn{19}{|c|}{$\beta$-Lactam/ $\beta$-Lactamase Inhibitor Combinations } \\
\hline Ampicillin-sulbactam & 0 & 0 & 0 & 0 & 3 & 100 & 0 & 0 & 0 & 0 & 0 & 0 & 0 & 0 & 0 & 0 & 0 & 0 \\
\hline Ampicillin-clavulanic acid & 0 & 0 & 0 & 0 & 0 & 0 & 0 & 0 & 0 & 0 & 0 & 0 & 0 & 0 & 0 & 0 & 0 & 0 \\
\hline Piperacillin-tazobactam & 0 & 0 & 1 & 20 & 3 & 100 & 0 & 0 & 4 & 50 & - & - & 0 & 0 & 2 & 100 & 0 & 0 \\
\hline \multicolumn{19}{|l|}{ Cephems } \\
\hline Cephalexin & 0 & 0 & 0 & 0 & 0 & 0 & 0 & 0 & 0 & 0 & 0 & 0 & 0 & 0 & 0 & 0 & 0 & 0 \\
\hline Cephradine & 0 & 0 & 0 & 0 & 0 & 0 & 0 & 0 & 0 & 0 & 0 & 0 & 0 & 0 & 0 & 0 & 0 & 0 \\
\hline Cefotaxime & 0 & 0 & 0 & 0 & 0 & 0 & 0 & 0 & 0 & 0 & 0 & 0 & 3 & 100 & 0 & 0 & 0 & 0 \\
\hline Cefoperazone & $2 / 15$ & 13.3 & 0 & 0 & 3 & 100 & 0 & 0 & 0 & 0 & 0 & 0 & - & - & 0 & 0 & 0 & 0 \\
\hline Cefquinome & 0 & 0 & 0 & 0 & 0 & 0 & 0 & 0 & 0 & 0 & 0 & 0 & 0 & 0 & 0 & 0 & 0 & 0 \\
\hline Monobactam & & & & & & & & & & & & & & & & & & \\
\hline Meropenem & $2 / 15$ & 13.3 & 0 & 0 & 0 & 0 & 0 & 0 & 0 & 0 & 0 & 0 & 3 & 100 & 0 & 0 & 0 & 0 \\
\hline Aztreonam & 0 & 0 & 5 & 100 & 0 & 0 & 0 & 0 & - & - & - & - & - & - & 0 & 0 & 0 & 0 \\
\hline Non $\beta$-lactam & & & & & & & & & & & & & & & & & & \\
\hline Macrolides & & & & & & & & & & & & & & & & & & \\
\hline Clarithromycin & 0 & 0 & 0 & 0 & 0 & 0 & 0 & 0 & 0 & 0 & 0 & 0 & 0 & 0 & 0 & 0 & 1 & 100 \\
\hline Erythromycin & 0 & 0 & 0 & 0 & 0 & 0 & 0 & 0 & 0 & 0 & 1 & 16.7 & 0 & 0 & 0 & 0 & 0 & 0 \\
\hline Tetracyclines & & & & & & & & & & & & & & & & & & \\
\hline Oxytetracycline & 0 & 0 & 0 & 0 & 0 & 0 & 1 & 100 & 0 & 0 & 0 & 0 & 0 & 0 & 0 & 0 & 0 & 0 \\
\hline Doxycycline & - & - & - & - & - & - & - & - & 0 & 0 & 1 & 16.7 & 3 & 100 & 0 & 0 & 0 & 0 \\
\hline Fluoroquinolones & & & & & & & & & & & & & & & & & & \\
\hline Norfloxacin & 0 & 0 & 0 & 0 & 1 & 33.3 & 0 & 0 & 0 & 0 & 0 & 0 & 0 & 0 & 0 & 0 & 0 & 0 \\
\hline Ofloxacin & 0 & 0 & 0 & 0 & 0 & 0 & 0 & 0 & 1 & 12.5 & 0 & 0 & 0 & 0 & 0 & 0 & 0 & 0 \\
\hline Lomefloxacin & $2 / 13$ & 15.4 & 0 & 0 & 0 & 0 & 0 & 0 & 2 & 25 & 0 & 0 & 0 & 0 & 2 & 100 & 0 & 0 \\
\hline Enrofloxacin & $1 / 15$ & 6.7 & 0 & 0 & 1 & 33.3 & 0 & 0 & $1-$ & - & - & - & - & - & - & - & 0 & 0 \\
\hline Aminoglycosides & & & & & & & & & & & & & & & & & & \\
\hline Kanamycin & 0 & 0 & 0 & 0 & 0 & 0 & 0 & 0 & 0 & 0 & 0 & 0 & 0 & 0 & 0 & 0 & 0 & 0 \\
\hline Phenicols & & & & & & & & & & & & & & & & & & \\
\hline chloramphenicol & $1 / 15$ & 6.7 & & & & & & & & & & & & & & & & \\
\hline Novobiocin & 0 & 0 & 0 & 0 & 0 & 0 & 0 & 0 & 0 & 0 & 0 & 0 & 0 & 0 & 0 & 0 & 0 & 0 \\
\hline Streptomycin & 0 & 0 & 0 & 0 & 0 & 0 & 0 & 0 & 0 & 0 & 0 & 0 & 0 & 0 & 0 & 0 & 0 & 0 \\
\hline Neomycin & $1 / 15$ & 6.7 & 0 & 0 & 0 & 0 & 0 & 0 & 0 & 0 & 0 & 0 & 0 & 0 & 0 & 0 & 0 & 0 \\
\hline Amikacin & 0 & 0 & 0 & 0 & 0 & 0 & 0 & 0 & 0 & 0 & 0 & 0 & 0 & 0 & 0 & 0 & 0 & 0 \\
\hline Oxazolidinones & & & & & & & & & & & & & & & & & & \\
\hline Linezolid & - & - & - & - & - & - & - & - & 0 & 0 & 0 & 0 & 0 & 0 & 0 & 0 & 0 & 0 \\
\hline Lincosamides & & & & & & & & & & & & & & & & & & \\
\hline Clindamycin & - & - & - & - & - & - & - & - & 0 & 0 & 0 & 0 & 0 & 0 & 0 & 0 & 0 & 0 \\
\hline Glycopeptides & & & & & & & & & & & & & & & & & & \\
\hline Vancomycin & - & - & - & - & - & - & - & - & 0 & 0 & 2 & 33.3 & 0 & 0 & 0 & 0 & 0 & 0 \\
\hline
\end{tabular}

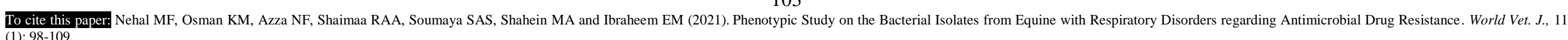


Table 8. Phenotypic resistance pattern of Gram-negative bacteria isolated from all samples.

\begin{tabular}{|c|c|c|c|c|c|c|c|c|c|c|c|c|c|c|c|c|c|c|c|c|c|c|c|c|c|c|}
\hline \multirow{2}{*}{ Bacterial isolates } & \multicolumn{3}{|c|}{$\beta$-lactam } & \multicolumn{3}{|c|}{ 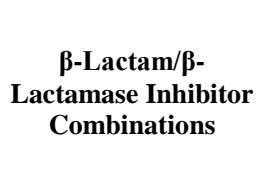 } & \multicolumn{5}{|c|}{ Cephems } & \multicolumn{2}{|c|}{ 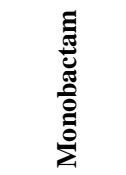 } & 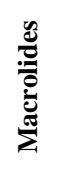 & \multicolumn{2}{|c|}{ 总 } & 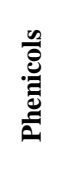 & \multicolumn{4}{|c|}{ Fluoroquinolones } & \multicolumn{5}{|c|}{ Aminoglycosides } \\
\hline & 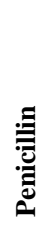 & 䔍 & 妾 & 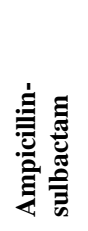 & 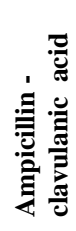 & 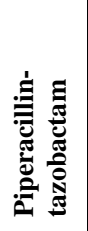 & 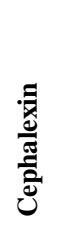 & Uू: & 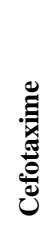 & Uूँ & 莺 & है: & 芘 & 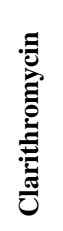 & 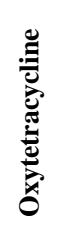 & 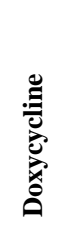 & 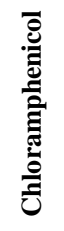 & 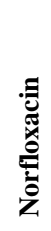 & 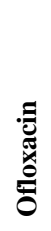 & 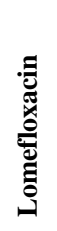 & 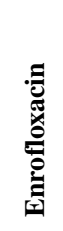 & 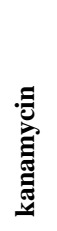 & 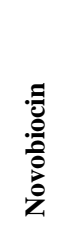 & 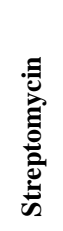 & 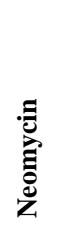 & 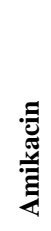 \\
\hline Klebsiella pneumoniae & $\mathrm{R}$ & & $\mathrm{R}$ & $\mathrm{R}$ & $\mathrm{R}$ & $\mathrm{R}$ & $\mathrm{R}$ & $\mathrm{R}$ & $\mathrm{R}$ & $S$ & $\mathrm{R}$ & $\mathrm{S}$ & $\mathrm{R}$ & $\mathrm{R}$ & $\mathrm{R}$ & & $\mathrm{S}$ & $\mathrm{R}$ & $\mathrm{R}$ & S & S & $\mathrm{R}$ & $\mathrm{R}$ & $\mathrm{R}$ & $\mathrm{S}$ & $\mathrm{R}$ \\
\hline Klebsiella pneumonia & $\mathrm{R}$ & & $\mathrm{R}$ & $\mathrm{R}$ & $\mathrm{R}$ & $\mathrm{R}$ & $\mathrm{R}$ & $\mathrm{R}$ & $\mathrm{R}$ & $\mathrm{S}$ & $\mathrm{R}$ & $\mathrm{S}$ & $\mathrm{R}$ & $\mathrm{R}$ & $\mathrm{R}$ & & $\mathrm{R}$ & $\mathrm{R}$ & $\mathrm{R}$ & $S$ & $\mathrm{R}$ & $\mathrm{R}$ & $\mathrm{R}$ & $\mathrm{R}$ & $\mathrm{R}$ & $\mathrm{R}$ \\
\hline Klebsiella pneumonia & $\mathrm{R}$ & & $\mathrm{R}$ & $\mathrm{R}$ & $\mathrm{R}$ & $\mathrm{R}$ & $\mathrm{R}$ & $\mathrm{R}$ & $\mathrm{R}$ & $\mathrm{R}$ & $\mathrm{R}$ & $\mathrm{R}$ & $\mathrm{R}$ & $\mathrm{R}$ & $\mathrm{R}$ & & $\mathrm{R}$ & $\mathrm{R}$ & $\mathrm{R}$ & $\mathrm{R}$ & $\mathrm{R}$ & $\mathrm{R}$ & $\mathrm{R}$ & $\mathrm{R}$ & $\mathrm{R}$ & $\mathrm{R}$ \\
\hline Klebsiella pneumonia & $\mathrm{R}$ & & $\mathrm{R}$ & $\mathrm{R}$ & $\mathrm{R}$ & $\mathrm{R}$ & $\mathrm{R}$ & $\mathrm{R}$ & $\mathrm{R}$ & $\mathrm{R}$ & $\mathrm{R}$ & $\mathrm{R}$ & $\mathrm{R}$ & $\mathrm{R}$ & $\mathrm{R}$ & & $\mathrm{R}$ & $\mathrm{R}$ & $\mathrm{R}$ & $\mathrm{R}$ & $\mathrm{R}$ & & $\mathrm{R}$ & $\mathrm{R}$ & $\mathrm{R}$ & $\mathrm{R}$ \\
\hline Klebsiella pneumonia & & & $\mathrm{R}$ & $\mathrm{R}$ & $\mathrm{R}$ & $\mathrm{R}$ & $\mathrm{R}$ & $\mathrm{R}$ & $\mathrm{R}$ & $\mathrm{R}$ & $\mathrm{R}$ & $\mathrm{R}$ & $\mathrm{R}$ & $\mathrm{R}$ & $\mathrm{R}$ & & $\mathrm{R}$ & $\mathrm{R}$ & $\mathrm{R}$ & $\mathrm{R}$ & $\mathrm{R}$ & & $\mathrm{R}$ & $\mathrm{R}$ & $\mathrm{R}$ & $\mathrm{R}$ \\
\hline Klebsiella pneumonia & & & $\mathrm{R}$ & $\mathrm{R}$ & $\mathrm{R}$ & $\mathrm{R}$ & $\mathrm{R}$ & $\mathrm{R}$ & $\mathrm{R}$ & $\mathrm{R}$ & $\mathrm{R}$ & $\mathrm{R}$ & $\mathrm{R}$ & $\mathrm{R}$ & $\mathrm{R}$ & & $\mathrm{R}$ & $\mathrm{R}$ & $\mathrm{R}$ & $\mathrm{R}$ & $\mathrm{R}$ & & $\mathrm{R}$ & $\mathrm{R}$ & $\mathrm{R}$ & $\mathrm{R}$ \\
\hline Klebsiella pneumonia & & & $\mathrm{R}$ & $\mathrm{R}$ & $\mathrm{R}$ & $\mathrm{R}$ & $\mathrm{R}$ & $\mathrm{R}$ & $\mathrm{R}$ & $\mathrm{R}$ & $\mathrm{R}$ & $\mathrm{R}$ & $\mathrm{R}$ & $\mathrm{R}$ & $\mathrm{R}$ & & $\mathrm{R}$ & $\mathrm{R}$ & $\mathrm{R}$ & $\mathrm{R}$ & $\mathrm{R}$ & & $\mathrm{R}$ & $\mathrm{R}$ & $\mathrm{R}$ & $\mathrm{R}$ \\
\hline Klebsiella pneumonia & & & $\mathrm{R}$ & $\mathrm{R}$ & $\mathrm{R}$ & $\mathrm{R}$ & $\mathrm{R}$ & $\mathrm{R}$ & $\mathrm{R}$ & $\mathrm{R}$ & $\mathrm{R}$ & $\mathrm{R}$ & $\mathrm{R}$ & $\mathrm{R}$ & $\mathrm{R}$ & & $\mathrm{R}$ & $\mathrm{R}$ & $\mathrm{R}$ & $\mathrm{R}$ & $\mathrm{R}$ & & $\mathrm{R}$ & $\mathrm{R}$ & $\mathrm{R}$ & $\mathrm{R}$ \\
\hline Klebsiella pneumonia & & & & $\mathrm{R}$ & $\mathrm{R}$ & $\mathrm{R}$ & $\mathrm{R}$ & $\mathrm{R}$ & $\mathrm{R}$ & $\mathrm{R}$ & $\mathrm{R}$ & $\mathrm{R}$ & $\mathrm{R}$ & $\mathrm{R}$ & $\mathrm{R}$ & & $\mathrm{R}$ & $\mathrm{R}$ & $\mathrm{R}$ & $\mathrm{R}$ & $\mathrm{R}$ & & $\mathrm{R}$ & $\mathrm{R}$ & $\mathrm{R}$ & $\mathrm{R}$ \\
\hline Klebsiella pneumonia & & & & $\mathrm{R}$ & $\mathrm{R}$ & $\mathrm{R}$ & $\mathrm{R}$ & $\mathrm{R}$ & $\mathrm{R}$ & $\mathrm{R}$ & $\mathrm{R}$ & $\mathrm{R}$ & $\mathrm{R}$ & $\mathrm{R}$ & $\mathrm{R}$ & & $\mathrm{R}$ & $\mathrm{R}$ & $\mathrm{R}$ & $\mathrm{R}$ & $\mathrm{R}$ & & $\mathrm{R}$ & $\mathrm{R}$ & $\mathrm{R}$ & $\mathrm{R}$ \\
\hline Klebsiella pneumonia & & & & $\mathrm{R}$ & $\mathrm{R}$ & $\mathrm{R}$ & $\mathrm{R}$ & $\mathrm{R}$ & $\mathrm{R}$ & $\mathrm{R}$ & $\mathrm{R}$ & $\mathrm{R}$ & $\mathrm{R}$ & $\mathrm{R}$ & $\mathrm{R}$ & & $\mathrm{R}$ & $\mathrm{R}$ & $\mathrm{R}$ & $\mathrm{R}$ & $\mathrm{R}$ & & $\mathrm{R}$ & $\mathrm{R}$ & $\mathrm{R}$ & $\mathrm{R}$ \\
\hline Klebsiella pneumonia & & & & $\mathrm{R}$ & $\mathrm{R}$ & $\mathrm{R}$ & $\mathrm{R}$ & $\mathrm{R}$ & $\mathrm{R}$ & $\mathrm{R}$ & $\mathrm{R}$ & $\mathrm{R}$ & $\mathrm{R}$ & $\mathrm{R}$ & $\mathrm{R}$ & & $\mathrm{R}$ & $\mathrm{R}$ & $\mathrm{R}$ & $\mathrm{R}$ & $\mathrm{R}$ & & $\mathrm{R}$ & $\mathrm{R}$ & $\mathrm{R}$ & $\mathrm{R}$ \\
\hline Klebsiella pneumonia & & & & $\mathrm{R}$ & $\mathrm{R}$ & $\mathrm{R}$ & $\mathrm{R}$ & $\mathrm{R}$ & $\mathrm{R}$ & $\mathrm{R}$ & $\mathrm{R}$ & $\mathrm{R}$ & $\mathrm{R}$ & $\mathrm{R}$ & $\mathrm{R}$ & & $\mathrm{R}$ & $\mathrm{R}$ & $\mathrm{R}$ & & $\mathrm{R}$ & & $\mathrm{R}$ & $\mathrm{R}$ & $\mathrm{R}$ & \\
\hline Klebsiella pneumonia & & & & $\mathrm{R}$ & $\mathrm{R}$ & $\mathrm{R}$ & $\mathrm{R}$ & $\mathrm{R}$ & $\mathrm{R}$ & $\mathrm{R}$ & $\mathrm{R}$ & $\mathrm{R}$ & $\mathrm{R}$ & $\mathrm{R}$ & $\mathrm{R}$ & & $\mathrm{R}$ & $\mathrm{R}$ & $\mathrm{R}$ & & $\mathrm{R}$ & & $\mathrm{R}$ & $\mathrm{R}$ & $\mathrm{R}$ & \\
\hline Klebsiella pneumonia & & & & $\mathrm{R}$ & & $\mathrm{R}$ & $\mathrm{R}$ & $\mathrm{R}$ & $\mathrm{R}$ & $\mathrm{R}$ & $\mathrm{R}$ & $\mathrm{R}$ & $\mathrm{R}$ & $\mathrm{R}$ & $\mathrm{R}$ & & $\mathrm{R}$ & $\mathrm{R}$ & $\mathrm{R}$ & & & & $\mathrm{R}$ & $\mathrm{R}$ & $\mathrm{R}$ & \\
\hline Pseudomonas aeruginosa & $\mathrm{R}$ & $\mathrm{R}$ & $\mathrm{R}$ & $\mathrm{R}$ & $\mathrm{R}$ & $S$ & $\mathrm{R}$ & $\mathrm{R}$ & $\mathrm{R}$ & $\mathrm{R}$ & $\mathrm{R}$ & $\mathrm{R}$ & $\mathrm{S}$ & $\mathrm{R}$ & $\mathrm{R}$ & & $\mathrm{R}$ & $\mathrm{R}$ & $\mathrm{R}$ & $\mathrm{R}$ & & $\mathrm{R}$ & $\mathrm{R}$ & $\mathrm{R}$ & $\mathrm{R}$ & $\mathrm{R}$ \\
\hline Pseudomonas aeruginosa & $\mathrm{R}$ & $\mathrm{R}$ & $\mathrm{R}$ & $\mathrm{R}$ & $\mathrm{R}$ & $\mathrm{R}$ & $\mathrm{R}$ & $\mathrm{R}$ & $\mathrm{R}$ & $\mathrm{R}$ & $\mathrm{R}$ & $\mathrm{R}$ & $\mathrm{S}$ & $\mathrm{R}$ & $\mathrm{R}$ & & $\mathrm{R}$ & $\mathrm{R}$ & $\mathrm{R}$ & $\mathrm{R}$ & & $\mathrm{R}$ & $\mathrm{R}$ & $\mathrm{R}$ & $\mathrm{R}$ & $\mathrm{R}$ \\
\hline Pseudomonas aeruginosa & $\mathrm{R}$ & $\mathrm{R}$ & $\mathrm{R}$ & $\mathrm{R}$ & $\mathrm{R}$ & $\mathrm{R}$ & $\mathrm{R}$ & $\mathrm{R}$ & $\mathrm{R}$ & $\mathrm{R}$ & $\mathrm{R}$ & $\mathrm{R}$ & $\mathrm{S}$ & $\mathrm{R}$ & $\mathrm{R}$ & & $\mathrm{R}$ & $\mathrm{R}$ & $\mathrm{R}$ & $\mathrm{R}$ & & $\mathrm{R}$ & $\mathrm{R}$ & $\mathrm{R}$ & $\mathrm{R}$ & $\mathrm{R}$ \\
\hline Pseudomonas aeruginosa & $\mathrm{R}$ & $\mathrm{R}$ & $\mathrm{R}$ & $\mathrm{R}$ & $\mathrm{R}$ & $\mathrm{R}$ & $\mathrm{R}$ & $\mathrm{R}$ & $\mathrm{R}$ & $\mathrm{R}$ & $\mathrm{R}$ & $\mathrm{R}$ & $\mathrm{S}$ & $\mathrm{R}$ & $\mathrm{R}$ & & $\mathrm{R}$ & $\mathrm{R}$ & $\mathrm{R}$ & $\mathrm{R}$ & & $\mathrm{R}$ & $\mathrm{R}$ & $\mathrm{R}$ & $\mathrm{R}$ & $\mathrm{R}$ \\
\hline Pseudomonas aeruginosa & $\mathrm{R}$ & $\mathrm{R}$ & $\mathrm{R}$ & $\mathrm{R}$ & $\mathrm{R}$ & $\mathrm{R}$ & $\mathrm{R}$ & $\mathrm{R}$ & $\mathrm{R}$ & $\mathrm{R}$ & $\mathrm{R}$ & $\mathrm{R}$ & $S$ & $\mathrm{R}$ & $\mathrm{R}$ & & $\mathrm{R}$ & $\mathrm{R}$ & $\mathrm{R}$ & $\mathrm{R}$ & & $\mathrm{R}$ & $\mathrm{R}$ & $\mathrm{R}$ & $\mathrm{R}$ & $\mathrm{R}$ \\
\hline Proteus mirabilis & $\mathrm{R}$ & $\mathrm{R}$ & $\mathrm{R}$ & $\mathrm{S}$ & $\mathrm{R}$ & $\mathrm{S}$ & $\mathrm{I}$ & $\mathrm{R}$ & $\mathrm{R}$ & $\mathrm{S}$ & $\mathrm{R}$ & $\mathrm{R}$ & $\mathrm{R}$ & $\mathrm{R}$ & $\mathrm{R}$ & & $\mathrm{R}$ & $\mathrm{S}$ & $\mathrm{R}$ & $\mathrm{R}$ & & $\mathrm{R}$ & $\mathrm{R}$ & $\mathrm{R}$ & $\mathrm{R}$ & $\mathrm{R}$ \\
\hline Proteus mirabilis & $\mathrm{R}$ & $\mathrm{R}$ & $\mathrm{R}$ & $\mathrm{S}$ & $\mathrm{R}$ & $\mathrm{S}$ & $\mathrm{I}$ & $\mathrm{R}$ & $\mathrm{R}$ & $\mathrm{S}$ & $\mathrm{R}$ & $\mathrm{R}$ & $\mathrm{R}$ & $\mathrm{R}$ & $\mathrm{R}$ & & $\mathrm{R}$ & $\mathrm{R}$ & $\mathrm{R}$ & $\mathrm{R}$ & & $\mathrm{R}$ & $\mathrm{R}$ & $\mathrm{R}$ & $\mathrm{R}$ & $\mathrm{R}$ \\
\hline Proteus mirabilis & $\mathrm{R}$ & $\mathrm{R}$ & $\mathrm{R}$ & $\mathrm{S}$ & $\mathrm{R}$ & $\mathrm{S}$ & $\mathrm{R}$ & $R$ & $R$ & $\mathrm{~S}$ & $R$ & $\mathrm{R}$ & $\mathrm{R}$ & $\mathrm{R}$ & R & & $\mathrm{R}$ & $\mathrm{R}$ & $\mathrm{R}$ & $\mathrm{R}$ & & $\mathrm{R}$ & $\mathrm{R}$ & $\mathrm{R}$ & $\mathrm{R}$ & $\mathrm{R}$ \\
\hline Stenotrophomonas. maltophilia & $\mathrm{R}$ & & & $\mathrm{R}$ & $\mathrm{R}$ & & & $\mathrm{R}$ & & & $\mathrm{R}$ & $\mathrm{R}$ & $\mathrm{R}$ & & $\mathrm{S}$ & & & & & $\mathrm{S}$ & & & & & & $\mathrm{R}$ \\
\hline
\end{tabular}

R: Resistant; S: Sensitive; I: intermediate. 
Table 9. phenotypic resistance pattern of Gram-positive bacteria isolated from different samples

\begin{tabular}{|c|c|c|c|c|c|c|c|c|c|c|c|c|c|c|c|c|c|c|c|c|c|c|c|c|c|c|c|c|}
\hline \multirow{2}{*}{ Bacterial isolates } & \multicolumn{3}{|c|}{$\beta$-lactam } & \multicolumn{3}{|c|}{$\begin{array}{c}\beta \text {-Lactam } / \boldsymbol{\beta}- \\
\text { Lactamase } \\
\text { Inhibitor } \\
\text { Combinations }\end{array}$} & \multicolumn{5}{|c|}{ Cephems } & 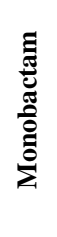 & \multicolumn{2}{|c|}{ 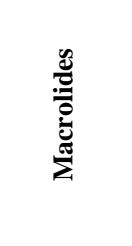 } & \multicolumn{2}{|c|}{ : } & \multirow{2}{*}{ 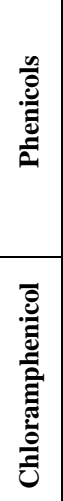 } & \multicolumn{3}{|c|}{ 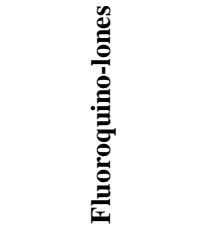 } & \multicolumn{5}{|c|}{ Aminoglycosides } & \multirow{2}{*}{ 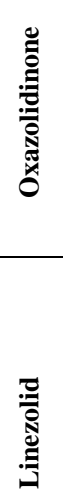 } & \multirow{2}{*}{ 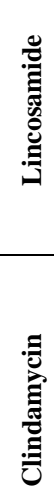 } & \multirow{2}{*}{ 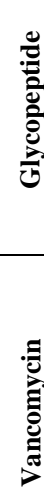 } \\
\hline & 多 & 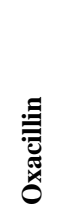 & 范 & 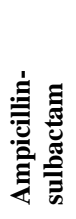 & 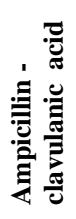 & 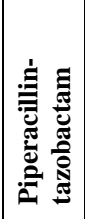 & 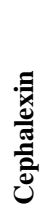 & 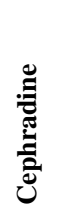 & 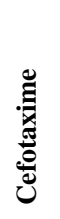 & 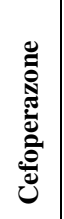 & 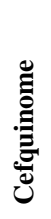 & & 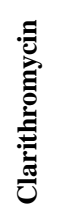 & 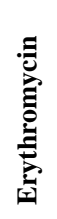 & 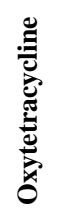 & 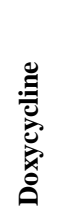 & & 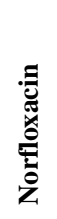 & 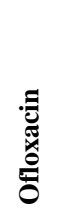 & 苛 & 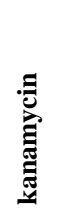 & 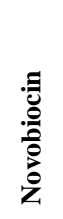 & 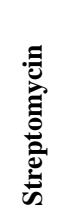 & 胥 & 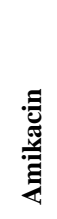 & & & \\
\hline Staphylococcus aureus & $\mathrm{R}$ & $\mathrm{R}$ & $\mathrm{R}$ & $\mathrm{R}$ & $\mathrm{R}$ & $\mathrm{S}$ & $\mathrm{R}$ & $\mathrm{R}$ & $\mathrm{R}$ & $\mathrm{R}$ & $\mathrm{R}$ & $\mathrm{R}$ & $\mathrm{R}$ & $\mathrm{R}$ & $\mathrm{R}$ & $\mathrm{R}$ & & $\mathrm{R}$ & $\mathrm{S}$ & $\mathrm{S}$ & $\mathrm{R}$ & $\mathrm{R}$ & $\mathrm{R}$ & $\mathrm{R}$ & $\mathrm{R}$ & $\mathrm{R}$ & $\mathrm{R}$ & $\mathrm{R}$ \\
\hline Staphylococcus aureus & $\mathrm{R}$ & $\mathrm{R}$ & $\mathrm{R}$ & $\mathrm{R}$ & $\mathrm{R}$ & $\mathrm{S}$ & $\mathrm{R}$ & $\mathrm{R}$ & $\mathrm{R}$ & $\mathrm{R}$ & $\mathrm{R}$ & $\mathrm{R}$ & $\mathrm{R}$ & $\mathrm{R}$ & $\mathrm{R}$ & $\mathrm{R}$ & & $\mathrm{R}$ & $\mathrm{R}$ & $\mathrm{S}$ & $\mathrm{R}$ & $\mathrm{R}$ & $\mathrm{R}$ & $\mathrm{R}$ & $\mathrm{R}$ & $\mathrm{R}$ & $\mathrm{R}$ & $\mathrm{R}$ \\
\hline Staphylococcus aureus & $\mathrm{R}$ & $\mathrm{R}$ & $\mathrm{R}$ & $\mathrm{R}$ & $\mathrm{R}$ & $S$ & $\mathrm{R}$ & & $\mathrm{R}$ & $\mathrm{R}$ & $\mathrm{R}$ & $\mathrm{R}$ & $\mathrm{R}$ & & $\mathrm{R}$ & $\mathrm{R}$ & & $\mathrm{R}$ & $\mathrm{R}$ & $\mathrm{R}$ & $\mathrm{R}$ & $\mathrm{R}$ & $\mathrm{R}$ & $\mathrm{R}$ & $\mathrm{R}$ & $\mathrm{R}$ & $\mathrm{R}$ & $\mathrm{R}$ \\
\hline Staphylococcus aureus & $\mathrm{R}$ & $\mathrm{R}$ & $\mathrm{R}$ & $\mathrm{R}$ & $\mathrm{R}$ & $\mathrm{S}$ & $\mathrm{R}$ & & $\mathrm{R}$ & $\mathrm{R}$ & $\mathrm{R}$ & $\mathrm{R}$ & $\mathrm{R}$ & & $\mathrm{R}$ & $\mathrm{R}$ & & $\mathrm{R}$ & $\mathrm{R}$ & $\mathrm{R}$ & $\mathrm{R}$ & $\mathrm{R}$ & $\mathrm{R}$ & $\mathrm{R}$ & $\mathrm{R}$ & $\mathrm{R}$ & $\mathrm{R}$ & $\mathrm{R}$ \\
\hline Staphylococcus aureus & $\mathrm{R}$ & $\mathrm{R}$ & $\mathrm{R}$ & $\mathrm{R}$ & $\mathrm{R}$ & $\mathrm{R}$ & $\mathrm{R}$ & & $\mathrm{R}$ & $\mathrm{R}$ & $\mathrm{R}$ & $\mathrm{R}$ & $\mathrm{R}$ & & $\mathrm{R}$ & $\mathrm{R}$ & & $\mathrm{R}$ & $\mathrm{R}$ & $\mathrm{R}$ & $\mathrm{R}$ & $\mathrm{R}$ & $\mathrm{R}$ & $\mathrm{R}$ & $\mathrm{R}$ & $\mathrm{R}$ & $\mathrm{R}$ & $\mathrm{R}$ \\
\hline Staphylococcus aureus & $\mathrm{R}$ & $\mathrm{R}$ & $\mathrm{R}$ & $\mathrm{R}$ & $\mathrm{R}$ & $\mathrm{R}$ & $\mathrm{R}$ & & $\mathrm{R}$ & $\mathrm{R}$ & $\mathrm{R}$ & $\mathrm{R}$ & $\mathrm{R}$ & & $\mathrm{R}$ & $\mathrm{R}$ & & $\mathrm{R}$ & $\mathrm{R}$ & $\mathrm{R}$ & $\mathrm{R}$ & $\mathrm{R}$ & $\mathrm{R}$ & $\mathrm{R}$ & $\mathrm{R}$ & $\mathrm{R}$ & $\mathrm{R}$ & $\mathrm{R}$ \\
\hline Staphylococcus aureus & $\mathrm{R}$ & $\mathrm{R}$ & $\mathrm{R}$ & $\mathrm{R}$ & $\mathrm{R}$ & $\mathrm{R}$ & $\mathrm{R}$ & & $\mathrm{R}$ & $\mathrm{R}$ & $\mathrm{R}$ & $\mathrm{R}$ & $\mathrm{R}$ & & $\mathrm{R}$ & $\mathrm{R}$ & & $\mathrm{R}$ & $\mathrm{R}$ & $\mathrm{R}$ & $\mathrm{R}$ & $\mathrm{R}$ & $\mathrm{R}$ & $\mathrm{R}$ & $\mathrm{R}$ & $\mathrm{R}$ & $\mathrm{R}$ & $\mathrm{R}$ \\
\hline Staphylococcus aureus & $\mathrm{R}$ & $\mathrm{R}$ & $\mathrm{R}$ & $\mathrm{R}$ & $\mathrm{R}$ & $\mathrm{R}$ & $\mathrm{R}$ & & $\mathrm{R}$ & $\mathrm{R}$ & $\mathrm{R}$ & $\mathrm{R}$ & $\mathrm{R}$ & & $\mathrm{R}$ & $\mathrm{R}$ & & $\mathrm{R}$ & $\mathrm{R}$ & $\mathrm{R}$ & $\mathrm{R}$ & $\mathrm{R}$ & $\mathrm{R}$ & $\mathrm{R}$ & $\mathrm{R}$ & $\mathrm{R}$ & $\mathrm{R}$ & $\mathrm{R}$ \\
\hline Streptococcus equi & $\mathrm{R}$ & $\mathrm{R}$ & $\mathrm{R}$ & $\mathrm{R}$ & $\mathrm{R}$ & & $\mathrm{R}$ & $\mathrm{R}$ & $\mathrm{R}$ & $\mathrm{R}$ & $\mathrm{R}$ & $\mathrm{S}$ & $\mathrm{R}$ & $\mathrm{R}$ & $\mathrm{R}$ & $\mathrm{S}$ & $\mathrm{R}$ & $\mathrm{R}$ & $\mathrm{R}$ & $\mathrm{R}$ & $\mathrm{R}$ & $\mathrm{R}$ & $\mathrm{R}$ & $\mathrm{R}$ & $\mathrm{R}$ & $\mathrm{S}$ & $\mathrm{R}$ & $\mathrm{S}$ \\
\hline Streptococcus equi & $\mathrm{R}$ & $\mathrm{R}$ & $\mathrm{R}$ & $\mathrm{R}$ & $\mathrm{R}$ & & $\mathrm{R}$ & $\mathrm{R}$ & $\mathrm{R}$ & $\mathrm{R}$ & $\mathrm{R}$ & $\mathrm{R}$ & $\mathrm{R}$ & $\mathrm{R}$ & $\mathrm{R}$ & $\mathrm{R}$ & $\mathrm{R}$ & $\mathrm{R}$ & $\mathrm{R}$ & $\mathrm{R}$ & $\mathrm{R}$ & $\mathrm{R}$ & $\mathrm{R}$ & $\mathrm{R}$ & $\mathrm{R}$ & $\mathrm{R}$ & $\mathrm{R}$ & $\mathrm{S}$ \\
\hline Streptococcus equi & $\mathrm{R}$ & $\mathrm{R}$ & $\mathrm{R}$ & $\mathrm{R}$ & $\mathrm{R}$ & & $\mathrm{R}$ & $\mathrm{R}$ & $\mathrm{R}$ & $\mathrm{R}$ & $\mathrm{R}$ & $\mathrm{R}$ & $\mathrm{R}$ & $\mathrm{R}$ & $\mathrm{R}$ & $\mathrm{R}$ & $\mathrm{R}$ & $\mathrm{R}$ & $\mathrm{R}$ & $\mathrm{R}$ & $\mathrm{R}$ & $\mathrm{R}$ & $\mathrm{R}$ & $\mathrm{R}$ & $\mathrm{R}$ & $\mathrm{R}$ & $\mathrm{R}$ & $\mathrm{R}$ \\
\hline Streptococcus equi & $\mathrm{R}$ & $\mathrm{R}$ & $\mathrm{R}$ & $\mathrm{R}$ & $\mathrm{R}$ & & $\mathrm{R}$ & $\mathrm{R}$ & $\mathrm{R}$ & $\mathrm{R}$ & $\mathrm{R}$ & $\mathrm{R}$ & $\mathrm{R}$ & $\mathrm{R}$ & $\mathrm{R}$ & $\mathrm{R}$ & $\mathrm{R}$ & $\mathrm{R}$ & $\mathrm{R}$ & $\mathrm{R}$ & $\mathrm{R}$ & $\mathrm{R}$ & $\mathrm{R}$ & $\mathrm{R}$ & $\mathrm{R}$ & $\mathrm{R}$ & $\mathrm{R}$ & $\mathrm{R}$ \\
\hline Streptococcus equi & $\mathrm{R}$ & $\mathrm{R}$ & $\mathrm{R}$ & $\mathrm{R}$ & $\mathrm{R}$ & & $\mathrm{R}$ & $\mathrm{R}$ & $\mathrm{R}$ & $\mathrm{R}$ & $\mathrm{R}$ & $\mathrm{R}$ & $\mathrm{R}$ & $\mathrm{R}$ & $\mathrm{R}$ & $\mathrm{R}$ & $\mathrm{R}$ & $\mathrm{R}$ & $\mathrm{R}$ & $\mathrm{R}$ & $\mathrm{R}$ & $\mathrm{R}$ & $\mathrm{R}$ & $\mathrm{R}$ & $\mathrm{R}$ & $\mathrm{R}$ & $\mathrm{R}$ & $\mathrm{R}$ \\
\hline Streptococcus equi & $\mathrm{R}$ & $\mathrm{R}$ & $\mathrm{R}$ & $\mathrm{R}$ & $\mathrm{R}$ & & $\mathrm{R}$ & $\mathrm{R}$ & $\mathrm{R}$ & $\mathrm{R}$ & $\mathrm{R}$ & $\mathrm{R}$ & $\mathrm{R}$ & $\mathrm{R}$ & $\mathrm{R}$ & $\mathrm{R}$ & $\mathrm{R}$ & $\mathrm{R}$ & $\mathrm{R}$ & $\mathrm{R}$ & $\mathrm{R}$ & $\mathrm{R}$ & $\mathrm{R}$ & $\mathrm{R}$ & $\mathrm{R}$ & $\mathrm{R}$ & $\mathrm{R}$ & $\mathrm{R}$ \\
\hline Streptococcus zooepidemicus & $\mathrm{R}$ & & $\mathrm{R}$ & $\mathrm{R}$ & $\mathrm{R}$ & & $\mathrm{R}$ & $\mathrm{R}$ & $\mathrm{S}$ & & $\mathrm{R}$ & $\mathrm{S}$ & $\mathrm{R}$ & $\mathrm{R}$ & $\mathrm{R}$ & $\mathrm{S}$ & & $\mathrm{R}$ & $\mathrm{R}$ & $\mathrm{R}$ & $\mathrm{R}$ & $\mathrm{R}$ & $\mathrm{R}$ & & $\mathrm{R}$ & & $\mathrm{R}$ & $\mathrm{R}$ \\
\hline Streptococcus zooepidemicus & $\mathrm{R}$ & & $\mathrm{R}$ & $\mathrm{R}$ & $\mathrm{R}$ & & $\mathrm{R}$ & $\mathrm{R}$ & S & & $\mathrm{R}$ & S & $\mathrm{R}$ & $\mathrm{R}$ & $\mathrm{R}$ & $\mathrm{S}$ & & $\mathrm{R}$ & $\mathrm{R}$ & $\mathrm{R}$ & $\mathrm{R}$ & $\mathrm{R}$ & $\mathrm{R}$ & & $\mathrm{R}$ & & $\mathrm{R}$ & $\mathrm{R}$ \\
\hline Streptococcus zooepidemicus & $\mathrm{R}$ & & $\mathrm{R}$ & $\mathrm{R}$ & $\mathrm{R}$ & & $\mathrm{R}$ & $\mathrm{R}$ & $\mathrm{S}$ & & $\mathrm{R}$ & $\mathrm{S}$ & $\mathbf{R}$ & $\mathbf{R}$ & $\mathbf{R}$ & $\mathbf{S}$ & & $\mathrm{R}$ & $\mathrm{R}$ & $\mathrm{I}$ & $\mathrm{R}$ & $\mathrm{R}$ & $\mathrm{R}$ & & $\mathrm{R}$ & & $\mathrm{R}$ & $\mathrm{R}$ \\
\hline Enterococcus species & $\mathrm{S}$ & $\mathrm{R}$ & $\mathrm{R}$ & $\mathrm{R}$ & $\mathrm{R}$ & $\mathrm{S}$ & $\mathrm{R}$ & $\mathrm{R}$ & $\mathrm{R}$ & $\mathrm{R}$ & $\mathrm{R}$ & $\mathrm{R}$ & $\mathrm{R}$ & $\mathrm{R}$ & $\mathrm{R}$ & $\mathrm{R}$ & & $\mathrm{R}$ & $\mathrm{R}$ & $\mathrm{S}$ & $\mathrm{R}$ & $\mathrm{R}$ & $\mathrm{R}$ & $\mathrm{R}$ & $\mathrm{R}$ & $\mathrm{R}$ & $\mathrm{R}$ & $\mathrm{R}$ \\
\hline Enterococcus species & $\mathrm{S}$ & $\mathrm{R}$ & $\mathrm{R}$ & $\mathrm{R}$ & $\mathrm{R}$ & $S$ & $\mathrm{R}$ & $\mathrm{R}$ & $\mathrm{R}$ & $\mathrm{R}$ & $\mathrm{R}$ & $\mathrm{R}$ & $\mathrm{R}$ & $\mathrm{R}$ & $\mathrm{R}$ & $\mathrm{R}$ & & $\mathrm{R}$ & $\mathrm{R}$ & $S$ & $\mathrm{R}$ & $\mathrm{R}$ & $\mathrm{R}$ & $\mathrm{R}$ & $\mathrm{R}$ & $\mathrm{R}$ & $\mathrm{R}$ & $\mathrm{R}$ \\
\hline Rhodococcus equi & $\mathrm{R}$ & $\mathrm{R}$ & $\mathrm{R}$ & $\mathrm{R}$ & $\mathrm{R}$ & $\mathrm{R}$ & $\mathrm{R}$ & $\mathrm{R}$ & $\mathrm{R}$ & $\mathrm{R}$ & $\mathrm{R}$ & $\mathrm{R}$ & $\mathrm{S}$ & $\mathrm{R}$ & $\mathrm{R}$ & $\mathrm{R}$ & $\mathrm{R}$ & $\mathrm{R}$ & $\mathrm{R}$ & $\mathrm{R}$ & $\mathrm{R}$ & $\mathrm{R}$ & $\mathrm{R}$ & $\mathrm{R}$ & $\mathrm{R}$ & $\mathrm{R}$ & $\mathrm{R}$ & $\mathrm{R}$ \\
\hline
\end{tabular}

R: Resistant, S.: Sensitive, I: intermediate 
Table 10. Multidrug resistance profiles of the Gram negative bacterial species isolated from respiratory tract of equines

\begin{tabular}{|c|c|c|c|c|c|}
\hline $\begin{array}{c}\text { Number of } \\
\text { resistant } \\
\text { AB }\end{array}$ & $\begin{array}{l}\text { Number of } \\
\text { resistant } \\
\text { AB classes }\end{array}$ & Antibiotics & $\begin{array}{c}\begin{array}{c}\text { Number } \\
\text { of } \\
\text { isolates }\end{array} \\
\end{array}$ & $\begin{array}{l}\text { Type of } \\
\text { resistance }\end{array}$ & $\begin{array}{c}\text { Total number } \\
\text { of Isolates } \\
(\mathbf{n}=\mathbf{2 4})\end{array}$ \\
\hline 9 & 5 & P, AMP, S, AMC, TZP, CL, CTX, CEQ, CLR & 1 & MDR & \multirow{9}{*}{$\begin{array}{c}15 \\
\text { (K.pneumoniae) }\end{array}$} \\
\hline 12 & 6 & P, AMP, AMC, TZP, CL, CTX, CEQ, C, ENR, NV, S, N & 1 & MDR & \\
\hline 16 & 8 & $\begin{array}{l}\text { P, AMP, AMC, TZP, CL, CTX, CEQ, MEM, ATM, CLR, C, LOM, ENR, } \\
\text { NV, S, N }\end{array}$ & 1 & MDR & \\
\hline 16 & 8 & $\begin{array}{l}\text { P, AMP, AMC, TZP, CL, CE, CEP CEQ, MEM, ATM, CLR, C, LOM, } \\
\text { ENR, NV, N }\end{array}$ & 1 & MDR & \\
\hline 18 & 8 & $\begin{array}{l}\text { SAM, TZP, CL, CE, CTX, CEP, CEQ, MEM, ATM, CLR, E, OT, C, NO, } \\
\text { OFX, NV, S, N }\end{array}$ & 1 & MDR & \\
\hline 20 & 8 & $\begin{array}{l}\text { SAM, AMC, TZP, CL, CE, CTX, CEP, CEQ, MEM, ATM, CLR, E, OT, } \\
\text { C, NO, OFX, ENR, NV, S, N }\end{array}$ & 2 & MDR & \\
\hline 20 & 8 & $\begin{array}{l}\text { SAM, AMC, TZP, CL, CE, CTX, CEP, CEQ, MEM, ATM, CLR, E, OT, } \\
\text { C, NO, OFX, ENR, NV, S, N }\end{array}$ & 2 & MDR & \\
\hline 22 & 8 & $\begin{array}{l}\text { SAM, AMC, TZP, CL, CE, CTX, CEP, CEQ, MEM, ATM, CLR, E, OT, } \\
\text { C, NO, OFX, LOM, ENR, NV, S, N, AK }\end{array}$ & 2 & MDR & \\
\hline 23 & 9 & $\begin{array}{l}\text { AMP, SAM, AMC, TZP, CL, CE, CTX, CEP, CEQ, MEM, ATM, CLR, } \\
\text { E, OT, C, NO, OFX, LOM, ENR, NV, S, N, AK }\end{array}$ & 4 & PDR & \\
\hline 23 & 9 & $\begin{array}{l}\text { P, OXA, AMP, SAM, AMC, TZP, CL, CE, CTX, CEP, CEQ, MEM, CLR, } \\
\text { OT, C, NO, OFX, LOM, K, NV, S, N, AK }\end{array}$ & 2 & PDR & \multirow{3}{*}{$\begin{array}{c}5 \\
(\text { P.aeruginosa })\end{array}$} \\
\hline 22 & 9 & $\begin{array}{l}\text { P, OXA, AMP, SAM, AMC, TZP, CL, CE, CTX, CEP, CEQ, MEM, CLR, } \\
\text { OT, C, NO, OFX, K, NV, S, N, AK }\end{array}$ & 2 & PDR & \\
\hline 21 & 9 & $\begin{array}{l}\text { P, OXA, AMP, SAM, AMC, CL, CE, CTX, CEP, CEQ, MEM, CLR, OT, } \\
\text { C, NO, OFX, K, NV, S, N, AK }\end{array}$ & 1 & PDR & \\
\hline 17 & 8 & $\begin{array}{l}\text { P, OXA, AMP, AMC, CE, CTX, CEQ, MEM, ATM, CLR, OT, C, K, NV, } \\
\text { S, N, AK }\end{array}$ & 1 & MDR & \multirow{2}{*}{$3(P$. mirabilis $)$} \\
\hline 16 & 8 & $\begin{array}{l}\text { P, OXA, AMP, AMC, CTX, CEQ, MEM, ATM, CLR, OT, C, K, NV, S, } \\
\text { N, AK }\end{array}$ & 2 & MDR & \\
\hline 6 & 8 & SAM, AMC, CE, CEQ, MEM, ATM & 1 & MDR & $\begin{array}{c}1 S . \\
\text { maltophilia) }\end{array}$ \\
\hline
\end{tabular}

P: Penicillin, OXA: Oxacillin, Amp: Ampicillin, SAM: Ampicillin-sulbactam, AMC: Ampicillin -clavulanic acid, PRL: Piperacillin-tazobactam, CFX: Cephalexin, CE: Cephradine, CTX: Cefotaxime, CPZ: Cefoperazone, CEQ: Cefquinome, MEM: Meropenem, ATM: Aztreonam, CLR: Clarithromycin, OXT: Oxytetracycline, C: Chloramphenicol, NOR: Norfloxacin, OFX: Ofloxacin, LOM: Lomefloxacin, ENR: Enrofloxacin, K: kanamycin, NO: Novobiocin, S: Streptomycin, N: Neomycin, AK: Amikacin, MDR: Multidrug resistant, PDR: Pan-drug resistant, n: Number, AB: Antibiotic.

Table 11. Multidrug resistance profiles of the Gram +ve bacteria species isolated from respiratory tract and feces of equines

\begin{tabular}{|c|c|c|c|c|c|}
\hline $\begin{array}{c}\text { Number of } \\
\text { resistant } \\
\text { AB } \\
\end{array}$ & $\begin{array}{c}\text { Number of } \\
\text { resistant } \\
\text { AB classes }\end{array}$ & Antibiotics & $\begin{array}{c}\begin{array}{c}\text { Number } \\
\text { of } \\
\text { isolates }\end{array} \\
\end{array}$ & $\begin{array}{l}\text { Type } \\
\text { of } \\
\text { AMR }\end{array}$ & $\begin{array}{c}\text { Number of } \\
\text { isolates } \\
(\mathbf{n}=19) \\
\end{array}$ \\
\hline 24 & 11 & $\begin{array}{l}\text { P, OXA, Amp, SAM, AMC, CFX, CE, CPZ, TZP, CEQ, MEM, CLR, } \\
\text { OXT, NOR, K, NV, DO, NO, S, N, AK, DA, VA, LZD }\end{array}$ & 1 & PDR & \multirow{5}{*}{$\begin{array}{c}8 \\
\text { (S. aureus) }\end{array}$} \\
\hline 25 & 11 & $\begin{array}{l}\text { P, OXA, Amp, SAM, AMC, CFX, CE, CPZ, TZP, CEQ, MEM, CLR, } \\
\text { OXT, NOR, OFX, K, NV, DO, NO, S, N, AK, DA, VA, LZD }\end{array}$ & 1 & PDR & \\
\hline 25 & 11 & $\begin{array}{l}\text { P, OXA, Amp, SAM, AMC, CFX, CPZ, TZP, CEQ, MEM, CLR, E, } \\
\text { OXT, NOR, OFX, LOM, NV, DO, NO, S, N, AK, DA, VA, LZD }\end{array}$ & 3 & PDR & \\
\hline 25 & 11 & $\begin{array}{l}\text { P, OXA, Amp, SAM, AMC, CFX, CPZ, TZP, CEQ, MEM, CLR, OXT, } \\
\text { NOR, OFX, LOM, K, NV, DO, NO, S, N, AK, DA, VA, LZD }\end{array}$ & 2 & PDR & \\
\hline 26 & 11 & $\begin{array}{l}\text { P, OXA, Amp, SAM, AMC, CFX, CPZ, TZP, CEQ, MEM, CLR, E, } \\
\text { OXT, NOR, OFX, LOM, K, NV, DO, NO, S, N, AK, DA, VA, LZD }\end{array}$ & 1 & PDR & \\
\hline 23 & 8 & $\begin{array}{l}\text { P, OXA, AMP, SAM, AMC, CE, CTX, KF, CEP, CEQ, CLR, E, OTX, } \\
\text { C, NOR, OFX, LOM, K, NV, S, N, AK, DA }\end{array}$ & 1 & MDR & \multirow{3}{*}{$\begin{array}{c}6 \\
\text { (S. equi Equi) }\end{array}$} \\
\hline 26 & 11 & $\begin{array}{l}\text { P, OXA, AMP, SAM, AMC, CE, CTX, KF, CEP, CEQ, MEM, CLR, E, } \\
\text { OTX, DO, C, NOR, OFX, LOM, K, NV, S, N, AK, LNZ, DA }\end{array}$ & 1 & PDR & \\
\hline 27 & 11 & $\begin{array}{l}\text { P, OXA, AMP, SAM, AMC, CE, CTX, KF, CEP, CEQ, MEM, CLR, E, } \\
\text { OTX, DO, C, NOR, OFX, LOM, K, NV, S, N, AK, LNZ, DA, VA }\end{array}$ & 4 & PDR & \\
\hline 18 & 9 & $\begin{array}{l}\text { P, AMP, SAM, AMC, CE, CF, CEQ, CLR, E, OTX, NOR, OFX, K, } \\
\text { NV, S, AK, DA, VA }\end{array}$ & 1 & MDR & \multirow{2}{*}{$\begin{array}{c}3 \mathrm{~S} . \\
\text { Zooepidemicus) }\end{array}$} \\
\hline 19 & 9 & $\begin{array}{l}\text { P, AMP, SAM, AMC, CE, CF, CEQ, CLR, E, OTX, NOR, OFX, LOM, } \\
\text { K, NV, S, AK, DA, VA }\end{array}$ & 2 & MDR & \\
\hline 24 & 11 & $\begin{array}{l}\text { OXA, AMP, SAM, AMC, CE, CTX, KF, CEP, CEQ, MEM, ATM, } \\
\text { CLR, E, OTX, DO, NOR, OFX, K, NV, N, AK, LNZ, DA, VA }\end{array}$ & 2 & PDR & $\begin{array}{c}2 \\
\text { (Enterococcus) }\end{array}$ \\
\hline 26 & 11 & $\begin{array}{l}\text { P, OXA, AMP, SAM, AMC, CE, CTX, KF, CEP, CEQ, MEM, E, } \\
\text { OTX, DO, C, NOR, OFX, LOM, K, NV, S, N, AK, LNZ, DA, VA }\end{array}$ & 1 & PDR & 1 R.equi \\
\hline
\end{tabular}

P: Penicillin, OXA: Oxacillin, Amp: Ampicillin, SAM: Ampicillin-sulbactam, AMC: Ampicillin-clavulanic acid, PRL: Piperacillin-tazobactam, CFX: Cephalexin, CE: Cephradine, CTX: Cefotaxime, CPZ: Cefoperazone, CEQ: Cefquinome, MEM: Meropenem, CLR: Clarithromycin, E: Erythromycin, OXT: Oxytetracycline, DO: Doxycycline, NOR: Norfloxacin, OFX: Ofloxacin, LOM: Lomefloxacin, K: kanamycin, NO: Novobiocin, S: Streptomycin, N: Neomycin, AK: Amikacin, LIN: Linezolid, DA: Clindamycin, VA: Vancomycin, MDR: Multidrug resistant, PDR: Pan-drug resistant, n: number, AB: Antibiotic 


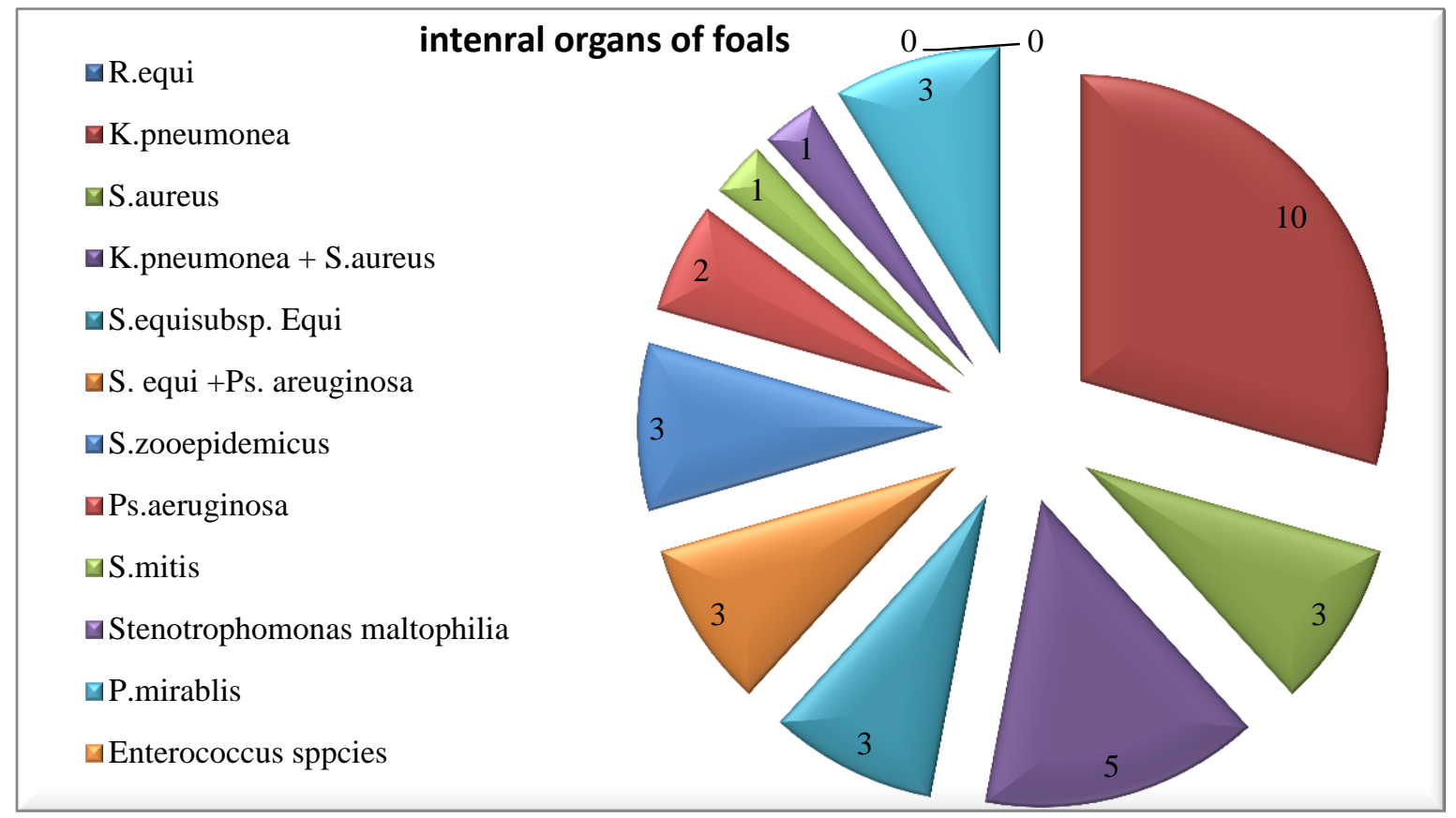

Figure 1. Number and type of isolates in internal organs of dead foals

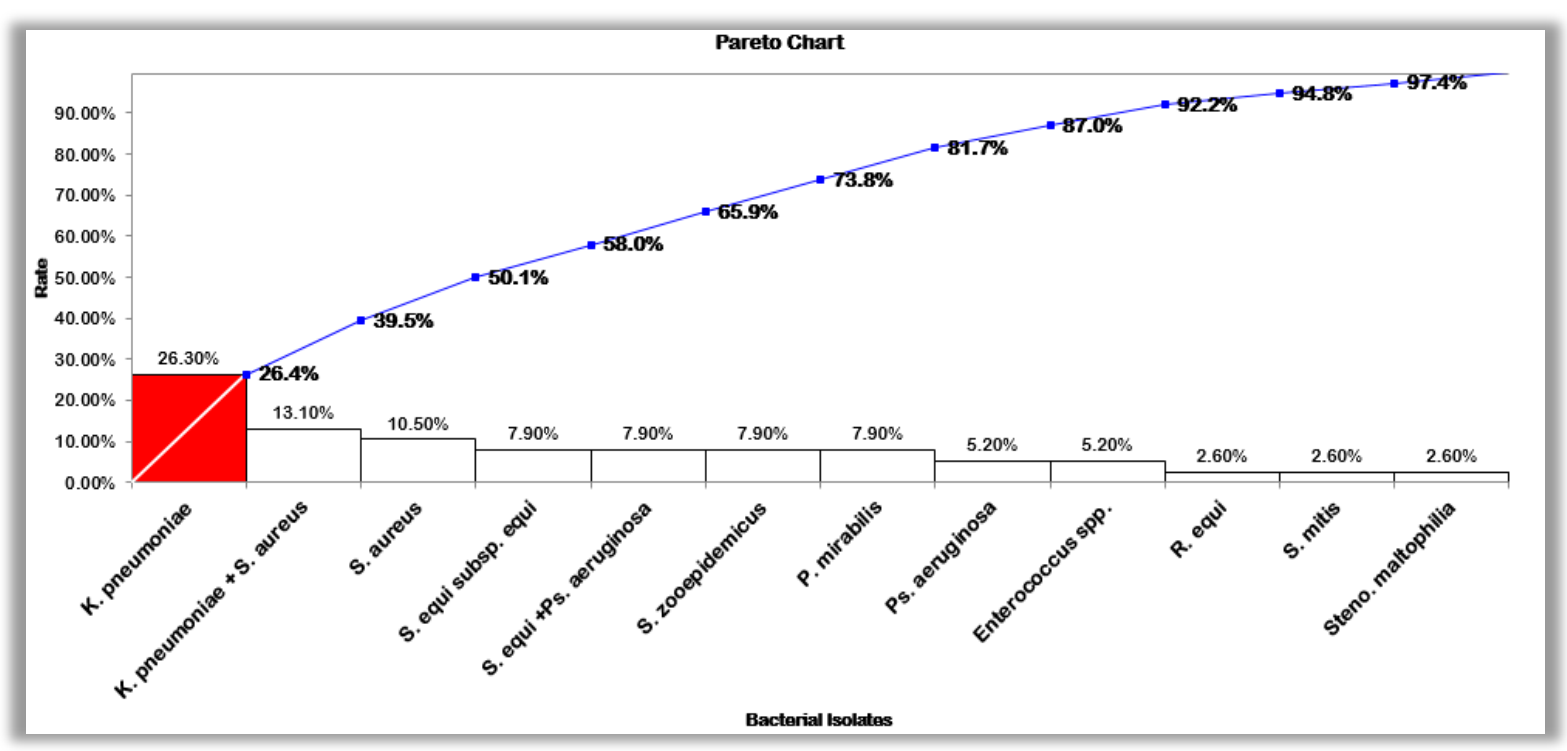

Figure 2. Pareto chart showing the rate of participation of different bacteria in respiratory infections in equine

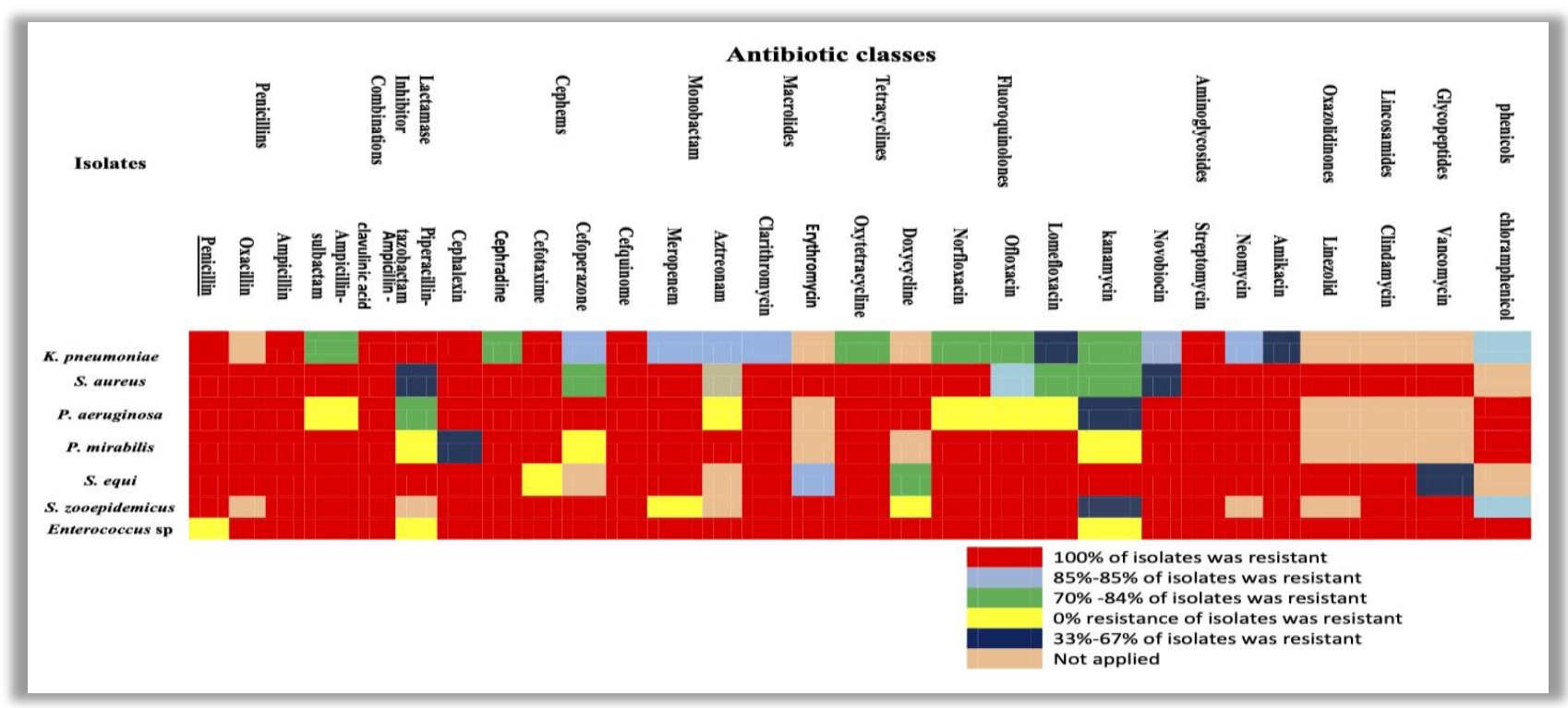

Figure 3. Heat map analysis showed the intensity of resistance of different isolates against different antimicrobial classes 


\title{
CONCLUSION
}

Stenotrophomonas maltophilia isolated from all organs, including the lung, is one of the first reports of isolation in Egypt. High rates of recorded antimicrobial resistance towards commonly used antibiotics emphasize the importance of individual bacteriological and antimicrobial susceptibility testing to guide antimicrobial therapy. The routine application of antimicrobials in the livestock industry has a dual effect, one acts as an advantage (beneficial for the health and productivity of the animal) while the other is considered as an important disadvantage with a global concern that is the significant evolution of different pathogenic bacterial strains having multidrug resistance (MDR) properties. In the present study, resistance monitoring data and risk assessment identified several direct and/or indirect predisposing factors to be potentially associated with MDR development in the equine health sector of Egypt. Affecting factors are inadequate veterinary healthcare, observing and controlling services, enhancing animal health knowledge among facility providers, and filling farmers' knowledge gap on drugs, and MDR which have resulted in the misuse and overuse of antibiotics leading to the evolution of antibiotic-resistant bacteria in equine in Egypt. Execution of extensive MDR, PDR, and XDR surveillance in equine and awareness programs for farmers along with the strengthening of the capacity of General Veterinary Services are recommended for effective containment of MDR emergence and spreading in the equine health sector in Egypt.

\section{DECLARATION}

\author{
Competing interests \\ Authors declare no conflict of interest.
}

\section{Authors' contributions}

Soumaya, S. A. El Shafii was responsible for project administration and validation. Nehal, M. Fawzy, Soumaya, S. A. El Shafii, Azza, N. F. and Shaimaa, R. A. Abd Elmawgoud cooperated in conceptualization, formal analysis, investigation, methodology, and writing the original draft. Kamelia, M. Osman, Momtaz A. Shahin, and Essam Ibrahim were helpful in data curation, writing, reviewing, and editing. All authors reviewed and approved the last edition of article for publishing in the present journal.

\section{Acknowledgments}

This paper is based upon work supported by Science, Technology \& Innovation Funding Authority (STDF) under the grant (Research Support Grant (STDF - RSG) / Capacity Building Grants).

\section{REFERENCES}

Álvarez-Narváez S, Berghaus LJ, Morris ERA, Willingham-Lane JM, Slovis NM, Giguere S, and Cohen ND (2020). A common practice of widespread antimicrobial use in horse production promotes multi-drug resistance. Scientific Reports, 10: Article number: 911 . Available at: https://www.doi.org/10.1038/s41598-020-57479-9

Boucher HW, Talbot GH, Bradley JS, Edwards JE, Gilbert D, Rice LB, Scheld M, Spellberg B, and Bartlett J (2009). Bad bugs, no drugs: no ESKAPE! An update from the infectious diseases society of America. Clinical Infectious Diseases, 48: 1-12.DOI: https://www.doi.org/10.1086/595011

Bousquet-Melou A, Bernard S, Schneider M, and Toutain PL (2002). Pharmacokinetics of marbofloxacin in horses. Equine Veterinary Journal, 34(4): 366-372. DOI: https://www.doi.org/10.2746/042516402776249191

Galvin NP and Corley KTT (2010). Causes of disease and death from birth to 12 months of age in the Thoroughbred horse in Ireland. Irish Veterinary Journal, 63(1): 37-43. https://www.doi.org/ 10.1186/2046-0481-63-1-37

Chirality (2012). Chirality in antibacterial agents. Comprehensive Chirality, pp. 30-53. DOI: https://www.doi.org/10.1016/B978-0-08-095167-6.00109$\underline{9}$

Clinical and Laboratory Standards Institute (CLSI) (2012). Performance Standards for Antimicrobial Susceptibility Testing. M07-A9. 32 (2): 15.

Clinical and Laboratory Standards Institute (CLSI) (2017). Clinical and Laboratory Standards Institute. Performance Standards for Antimicrobial Susceptibility Testing. M100-S26, 27th ed, p. 32, 52, 54. http://file.qums.ac.ir/repository/mmrc/clsi\%202017.pdf

Coates A, Hu Y, Bax R, and Page C (2002). The future challenges facing the development of new antimicrobial drugs. Nature Reviews Drug Discovery, 1: 895-910. DOI: https://www.doi.org/10.1038/nrd940

Davis JL, Papich MG, and Weingarten A (2006). The pharmacokinetics of orbifloxacin in the horse following oral and intravenous administration. Journal of Veterinary Pharmacology and Therapeutics, 29(3): 191-197. DOI: https://www.doi.org/10.1111/j.1365-2885.2006.00737.x

Deredjian A, Alliot N, Blanchard L, Brothier E, Anane M, Cambier P, Jolivet C, Khelil MN, Nazaret S, Saby N et al. (2016). Occurrence of Stenotrophomonas maltophilia in agricultural soils and antibiotic resistance properties. Research in Microbiology, 167(4): 313-324. DOI: https://www.doi.org/10.1016/j.resmic.2016.01.001

El Zowalaty ME, Al Thani AA, Webster TJ, El Zowalaty AE, Schweizer HP, Nasrallah GK, Marei HE, Ashour HM. (2015). Pseudomonas aeruginosa: arsenal of resistance mechanisms, decades of changing resistance profiles, and future antimicrobial therapies. Future Microbiology. 10(10):1683-706. https://www.doi.org/10.2217/fmb.15.48

Erol E, Locke SJ, Donahoe JK, Mackin MA, and Carter CN (2012). Beta-hemolytic Streptococcus spp. from horses: A retrospective study (20002010). Journal of Veterinary Diagnostic Investigation, 24(1): 142-147. DOI: https://www.doi.org/10.1177/1040638711434138

Estell KE, Young A, Kozikowski T, Swain EA, Byrne BA, Reilly CM, Kass PH, and Aleman M (2016). Pneumonia Caused by Klebsiella spp. in 46 Horses. Journal of Veterinary Internal Medicine, 30(1): 314-321. DOI: https://www.doi.org/10.1111/jvim.13653

Fernandez-Varon E, Ayala I, Marín P, Carrión A, Martos N, Escudero E, and Cárceles CM (2006). Pharmacokinetics of danofloxacin in horses after intravenous, intramuscular and intragastric administration. Equine Veterinary Journal, 38(4): 342-346. 
Fodor A, Abate BA, Péter Deák P, László Fodor L, Gyenge E, Michael G, Klein Koncz Z, Muvevi J, Ötvös L et al. (2020). Multidrug resistance (MDR) and collateral sensitivity in bacteria, with special attention to genetic and evolutionary aspects and to the perspectives of antimicrobial peptides - a review. Pathogens, 9: 522. DOI: https://www.doi.org/10.3390/pathogens9070522

Garza-Cervantes JA, Meza-Bustillos JF, Resendiz-Hernández H, Suárez-Cantú IA, Ortega-Rivera OA, Salinas E, Escárcega-González CE, and Morones-Ramírez JR (2020). Re-sensitizing ampicillin and kanamycin-resistant E. coli and S. aureus using synergistic metal micronutrientsantibiotic combinations. Frontiers in Bioengineering and Biotechnology, 8: 612. DOI: https://www.doi.org/10.3389/fbioe.2020.00612

Gigue`re S, Cohen ND, Keith Chaffin M, Slovis NM, Hondalus MK, Hines SA, and Prescott JF (2011). Diagnosis, treatment, control, and prevention of infections caused by Rhodococcus equi in foals. Journal of Veterinary Internal Medicine, 25: 1209-1220. DOI: https://www.doi.org/10.1111/j.1939-1676.2011.00835.x

Haggett EF, and Wilson WD (2008). Overview of the use of antimicrobials for the treatment of bacterial infections in horses. Equine Veterinary Education, 20(8): 433-448. DOI: https://www.doi.org/10.2746/095777308X338893

Hegreness M, Shoresh N, Damian D, Hartl D, and Kishony R (2008). Accelerated evolution of resistance in multidrug environments. Proceedings of the National Academy of Sciences, 105: 13977-13981. DOI: https://www.doi.org/10.1073/pnas.0805965105

https://www.doi.org/10.2746/042516406777749245

Johns IC, and Adams EL (2015). Trends in antimicrobial resistance in equine bacterial isolates: 1999-2012. Veterinary Record, 176(13): 334-334. DOI: https://www.doi.org/10.1136/vr.102708

Léguillette R, Roy MF, and Lavoie JP (2002). Foal pneumonia in: Equine respiratory diseases. International Veterinary Information Service, Ithaca NY. Available at: https://www.ivis.org/library/equine-respiratory-diseases

Lemmen SW, Zolldann D, Klik S, Lütticken R, Kümmerer K, and Häfner H (2004). Serum bactericidal activity of piperacillin/tazobactam against Staphylococcus aureus, piperacillin-susceptible and piperacillin-resistant Escherichia coli and Pseudomonas aeruginosa. Chemotherapy, 50(1): 27-30. DOI: https://www.doi.org/10.1159/000077281

Lönker NS, Fechner K, and Abd El Wahed A (2020). Horses as a Crucial Part of One Health. Veterinary Sciences ,7: Article number: 28. DOI: https://www.doi.org/10.3390/vetsci7010028

Magiorakos AP, Srinivasan A, Carey RB, Carmeli Y, Falagas ME, Giske CG, Harbarth S, Hindler JF, Kahlmeter G, Olsson-Liljequist B et al. (2012). Multidrug-resistant, extensively drug-resistant and pandrugresistant bacteria: An international expert proposal for interim standard definitions for acquired resistance. Clinical Microbiology and Infection, 18: 268-281. DOI: https://www.doi.org/10.1111/j.1469-0691.2011.03570.x

O'Hara CM, Brenner FW, and Miller JM (2000). Classification, identification, and clinical significance of Proteus, Providencia, and Morganella. Clinical Microbiology Reviews, 13(4): 534-546. DOI: https://www.doi.org/10.1128/cmr.13.4.534-546.2000

Pendleton JN, Gorman SP, and Gilmore BF (2013). Clinical relevance of the ESKAPE pathogens. Expert Review of Anti-infective Therapy, 11: 297308. DOI: https://www.doi.org/10.1586/eri.13.12

Roberts MC (2005). Update on acquired tetracycline resistance genes. FEMS Microbiology Letters, 245(2): 195-203. DOI: https://www.doi.org/10.1016/j.femsle.2005.02.034

Rush BR (2014). Strangles in Horses (Distemper). MSD and the MSD Veterinary Manual Merck \& Co., Inc., Kenilworth, NJ, USA. Available at: https://www.msdvetmanual.com/respiratory-system/respiratory-diseases-of-horses/strangles-in-horses

Saastamoinen M, Särkijärvi S, and Hyyppä S (2015). Reducing respiratory health risks to horses and workers: A comparison of two stall bedding materials. Animals, 5(4): 965-977. DOI: https://www.doi.org/10.3390/ani5040394

Smith PA and Romesberg FE (2007). Combating bacteria and drug resistance by inhibiting mechanisms of persistence and adaptation. Nature Chemical Biology, 3: 549-556. Avaible: https://www.doi.org/10.1038/nchembio.2007.27

Toombs-Ruane LJ, Riley CB, Kendall AT, Hill KE, Benschop J, and Rosanowski SM (2015). Antimicrobial susceptibility of bacteria isolated from neonatal foal samples submitted to a New Zealand veterinary pathology laboratory (2004 to 2013). New Zealand veterinary journal, 64(2): 107111. DOI: https://www.doi.org/10.1080/00480169.2015.1109006

UK standard (2014a). Standards for Microbiology Investigations ID 4 identification of Streptococcus species, Enterococcus species and morphologically similar organisms. UK Standards for Microbiology Investigations | Issued by the Standards Unit, Public Health England. Bacteriology Identification, $\quad$ ID, 4(3): Availabe https://assets.publishing.service.gov.uk/government/uploads/system/uploads/attachment_data/file/369827/ID_4i1_RUC_October_2014.pdf

UK standard (2014b). UK Standards for Microbiology Investigations for identification of Staphylococcus species, Micrococcus species and Rothia species. UK Standards for Microbiology Investigations | Issued by the Standards Unit, Public Health England.UK, 4: 1-26. Availalbe at: https://assets.publishing.service.gov.uk/government/uploads/system/uploads/attachment_data/file/887570/UK_SMI_ID_07i4.pdf

UK standard (2014c). UK Standards for Microbiology Investigations: identification of Corynebacterium species. UK Standards for Microbiology Investigations | Issued by the Standards Unit, Public Health England. Bacteriology - Identification, 4.1: 1-24. Available at: https://assets.publishing.service.gov.uk/government/uploads/system/uploads/attachment data/file/371490/ID 2i4.1.pdf

UK standard (2014d). UK Standards for Microbiology Investigations identification of Enterobacteriaceae. UK Standards for Microbiology Investigations | Issued by the Standards Unit, Public Health England. Bacteriology - Identification, 4: 1-34 Available at: https://assets.publishing.service.gov.uk/government/uploads/system/uploads/attachment_data/file/423601/ID_16i4.pdf

UK standard (2015). UK Standards for Microbiology Investigations Identification of Pseudomonas species and other Non Glucose Fermenters. UK Standards for Microbiology Investigations | Issued by the Standards Unit, Public Health England. Bacteriology - Identification, 3: 1-41. Available at: https://assets.publishing.service.gov.uk/government/uploads/system/uploads/attachment data/file/422699/ID 17i3.pdf

Welsh RD (1984). The significance of Streptococcus zooepidemicus in the horse. Equine Practice, 6: 6-16.

Wilson W (2001). Rational selection of antimicrobials for use in horses. In PROCEEDINGS AAEP, 47: 75-93. Available at: https://cmapspublic3.ihmc.us/rid=1NBG7LTJ4-52R6971Y03/Rational\%20Selection\%20of\%20Antimicrobials\%20for\%20Use\%20in\%20Horses.pdf

Winther L, Andersen RM, Baptiste KE, Aalbæk B, and Guardabassi L (2009). Association of Stenotrophomonas maltophilia infection with lower airway disease in the horse: a retrospective case series. The Veterinary Journal, 186(3): 358-363. DOI: https://www.doi.org/10.1016/j.tvj1.2009.08.026

Wolska KI, Grzes K, and Kurek K (2012). Synergy between novel antimicrobials and conventional antibiotics or bacteriocins. Polish Journal of Microbiology, 61: 95-104. DOI: https://www.doi.org/10.33073/pjm-2012-012

Wood JL, Newton JR, Chanter N, and Mumford JA (2005). Association between respiratory disease and bacterial and viral infections in British racehorses. Journal of Clinical Microbiology, 43: 120-126. DOI: https://www.doi.org/10.1128/JCM.43.1.120-126.2005 